\title{
Verbreitung und Laichverhältnisse von Sardelle und Sardine in der südöstlichen Nordsee und ihre Veränderungen als Folge der Klimaänderung
}

\author{
Von Horst Joachim Aurich \\ Biologische Anstalt Helgoland, List auf Sylt \\ Forschungsinstitut der Bundesanstalt für Fischerei
}

(Mit 7 Abildungen im Text)

Inhaltsübersicht

\begin{abstract}
1. Bedeutung der Untersuchungen, Seite 175 - II. Arbeitsmethode, S. 176 -- III. Die Sardelle, Engraulis encrasicholus L., S. 177 - 1. Allgemeine Verbreitung, S. $177-2$. Seit 1930 eingetretene Veränderungen, S. $178-3$. Rassen-Zugehörigkeit, S. $179-4$. Laichverhältnisse vor 1948, S. $180-5$. Laichverhältnisse seit 1948, S. $181-6$. Folgerungen aus der Verteilung der Sardellen-Brut, S. 189 - IV. Die Sardine, Clupea pilchardus Walbaum, S. 191 - 1. Allgemeine Verbreitung, S. 191 - 2. Laichverhältnisse, S. 192 - 3. Folgerungen aus der Verteilung der Sardinen-Brut, S. 194 - V. Diskussion der Ergebnisse, S. 195 - 1. Massenwechsel, S. $195-2$ - Beziehungen zur Klimaänderung, S. 200 - VI. Zusammenfassung, S. 202.
\end{abstract}

\section{Bedeutung der Untersuchuungen}

Als nach dem vergangenen Kriege wieder Forschungsfahrten auf See unternommen werden konnten, ergab sich, daß in der Fauna der südlichen Nordsee bedeutende Veränderungen eingetreten waren. Besonders auffallend war die ungewöhnlich starke Zunahme der Brutdichte bei einigen mediterranatlantischen Fischarten sowie das Vorkommen von Sardinen-Laich in der südlichen Nordsee.

Es besteht kein Zweifel, daß diese Beobachtungen in engem Zusammenhang mit der seit mehreren Jahrzehnten nachweisbaren Klimaänderung stehen, die gerade in den letzten Jahren bedeutsame Folgen für die praktische Fischerei gehabt hat: Rückgang der Erträge auf den bisherigen Fangplätzen, Verlagerung der Fanggebiete nach Norden, Umstellung der Fischerei auf andere Arten. Eine ausführliche Untersuchung aller mit der Klimaänderung in Zusammenhang stehenden biologischen Veränderungen wird daher von besonderem Wert für die angewandte Meeresforschung, die Fischereiwissenschaft, sein, die vor allem die Fragen interessieren: Verläuft die Änderung der klimatischen Faktoren und damit auch die Verlagerungen und der Massenwechsel der Fischbestände im gleichen Sinne noch weiter, wie lange noch, und welche Folgen wird eine Umkehr dieser Entwicklung haben? Aber auch der rein ökologischen Forschung 
bietet sich hier eine wertvolle Gelegenheit, gleichsam wie in einem groß angelegten Experiment unmittelbaren Einblick in die großen, das Maß normaler Schwankungen überschreitenden Verschiebungen der Verbreitungsareale, der Wanderstraßen und Laichgebiete sowie in den Massenwechsel der Meeresorganismen zu gewinnen.

Eine Deutung dieses gesamten, durch die Klimaänderung ausgelösten Geschehens und die Aufklärung der kausalen Zusammenhänge wird aber nur dann möglich sein, wenn den meteorologischen und hydrographischen Beobachtungsreihen ebensolche über die biologischen Vorgänge gegenübergestellt werden können. Das erfordert eine fortlaufende Kontrolle der biologischen Verhältnisse, möglichst auf quantitativer, zahlenmäßig vergleichbarer Basis.

In diesem Sinne wurden von der Biologischen Anstalt Helgoland die bereits 1902 begonnenen Plankton- und Fischbrutuntersuchungen in der südlichen Nordsee auch nach dem Kriege fortgeführt. In der vorliegenden Mitteilung wird zunächst nur über die Bestandsveränderungen bei der Sardelle und Sardine berichtet werden, bei denen die Beziehungen zu den klimatischen Veränderungen besonders deutlich sind.

Bei der Materialsammlung an Bord des Forschungskutters "Uthörn" erfuhr ich treue Hilfe von seiten der Kapitäne, Herrn Fischmeister J. HoltMann und seines Nachfolgers Herrn T. Hornsmann, sowie der Schiffsbesatzung, denen ich auch an dieser Stelle herzlich danke. An der weiteren Aufarbeitung und am Aussuchen der Fänge beteiligten sich Studenten, für deren Hilfe von der Deutschen Wissenschaftlichen Kommission für Meeresforschung ein finanzieller Beitrag dankenswerterweise gewährt wurde.

\section{Arbeitsmethode}

Zur Beurteilung der Bestandsdichten bedienen wir uns der von Hensen eingeführten Methode der Bestimmung der Zahl der unter der Flächeneinheit vorhandenen, planktischen Eier und Larven mittels Vertikalfängen mit dem Hensenschen Eiernetz (weitere Angaben über Fangtechnik, Bearbeitung und Auswertung der Fänge bei Bückmann 1929, Mielk \& Künne 1935). Diese Methode hat den großen Vorzug, objektive, zahlenmäßig vergleichbare Werte zu liefern, die in unmittelbarer Beziehung zur Zahl der laichenden Fische stehen; sie ist ferner unabhängig von Störungen der vertikalen Schichtung des Planktons durch Turbulenz und Strömungen, die starken Einfluß auf die aus Horizontal-Fängen gewonnenen Ergebnisse haben können. Gegenüber einer Beurteilung der Bestandsgröße aus den Fangerträgen ausgewachsener Fische haben die Fischbrutuntersuchungen außerdem den wesentlichen Vorteil, weder durch Faktoren politischer oder wirtschaftlicher Art (Fangbeschränkungen, Nachfrage auf dem Markt, Preisspekulationen) noch durch solche der Fangtechnik (unterschiedliche Fängigkeit verschiedener Fanggeschirre, Maschinenstärke und Größe der Schiffe) beeinflußt zu werden. Es ist daher empfehlenswert, auch in Zukunft an der alten Hensenschen Methode festzuhalten.

Im folgenden wird als Maß für die Brutdichte stets die Zahl der Eier oder Larven unter 1 Quadratmeter Meeresoberfläche angegeben. In den Verbreitungskarten werden diese Werte durch Rechtecke entsprechenden Flächeninhalts, bei engerem Stationsnetz auch in Form von Isoplankten, wiedergegeben. 
III. Die Sardelle, Engraulis encrasicholus L.

1. Allgemeine Verbreitung

Tiergeographisch gesehen, gehört die Sardelle zu den mediterran-atlantischen Formen, die, wenigstens zur Zeit ihrer Fortpflanzung und Larvenentwicklung, höhere Temperaturansprüche stellen. Das Hauptgebiet ihrer Verbreitung ist das Mittelmeer und Schwarze Meer sowie das atlantische Küstengebiet Südwest-Europas und Nord-Afrikas. In diesen Gebieten ist die Sardelle das ganze Jahr über anzutreffen, und hier liegen auch ihre hauptsächlichsten Fangplätze. Nach Süden erstreckt sich ihr Vorkommen bis vor den Südeingang des Suez-Kanals und längs der Westküste Afrikas über die Kanarischen Inseln bis Senegal, Togo und Dahomey. Im Norden reicht es durch die Biskaya-See, wo junge Sardellen nach Funden in den Mägen des Weißen Thuns (Germo alalonga) seewärts bis $11^{0} \mathrm{~W}$ vorkommen (LEGENDRE 1934), bis vor den Westeingang des Englischen Kanals. Hier wird die Nordgrenze ihres ständigen Wohngebietes erreicht.

Nach den Untersuchungen von Marion, Gourret, Holt und besonders FAGE (1911) ist die Sardelle in diesen Gebieten, zumindest im Mittelmeer, nur in engeren Grenzen ein Wanderfisch. Sie erscheint im Frühjahr und Sommer, zu großen Schwärmen vereinigt, sowohl auf hoher See wie auch an der Küste im Oberflächenwasser, führt in dieser Zeit ein rein pelagisches Dasein und schreitet im Mai, gelegentlich auch schon im April, zum Laichen, das sich bis in den September hinzieht. Diese Monate sind auch die Zeit der praktischen Fischerei auf Sardellen. Nach dem Ablaichen und mit Beginn des Winters ziehen sich die ausgelaichten Tiere mit den inzwischen aus den im Frühjahr und Sommer abgelegten Eiern herangewachsenen Jungfischen wieder in die Tiefe $(100$ bis $150 \mathrm{~m}$ ) zurück. Dort gehen sie, wie aus Untersuchungen ihres Mageninhalts zu schließen ist, zu einem mehr stationären Leben in Bodennähe über. Vermutlich lösen sich in dieser Zeit die Schwärme mehr oder weniger auf, da während des Winters Sardellen nur noch ganz vereinzelt gefangen werden. Die Nachkommen aus dem Herbstlaich verbleiben dagegen auch den Winter über im Oberflächenwasser der Küste und reifen hier bis zum folgenden Frühjahr heran.

Anders verhält sich die Sardelle in den nördlichen Randgebieten. Von hier dringt sie alljährlich als ausgesprochener Wanderfisch in großen Schwärmen zum Laichen weiter nach Norden und Nordosten vor. Ein Teil wandert in den Bristol-Kanal und in die Irische See ein, wo Sardellen von Mai bis September bis vor der schottischen Westküste auftreten, ohne jedoch eine lohnende Fischerei zu ermöglichen. Laichreife Weibchen und Eier wurden im Juni vor der Lancashire-Küste beobachtet (Cunningham; Jenkins; MaC InTosh \& MASTERMAN).

Weitaus bedeutender ist dagegen die Einwanderung in die Nordsee. Sie erfolgt im zeitigen Frühjahr durch den Englischen Kanal, und zwar in dessen südlicher Hälfte, da in dieser Zeit vor der englischen Küste Sardellen nur ausnahmsweise einmal beobachtet werden. Новк (1912) führt dies auf die besondere Temperatur-Verteilung im Kanal während des April zurück, zu welcher Zeit die Temperaturen an der französisch-belgischen Küste stets höher liegen als an den gegenüberliegenden Punkten der englischen Küste. Auf dieser Wanderung bleiben Teilschwärme vor der Seine- und Schelde-Mündung zurück, die Masse gelangt jedoch bis in die Zuider Zee und vor die ostfriesische 
Küste, wo sie eine zum Teil beachtliche Fischerei ermöglicht. In diesen Gebieten lagen bisher die nordöstlichsten Laichplätze der Sardelle.

Vereinzelt wandern Sardellen noch weiter, teils an die Ostküste Englands und Schottlands (The Wash, Firth of Forth, Aberdeen, Moray Firth), teils an die Küsten Jütlands und Norwegens (von Oslo bis Bergen), selten sogar bis ins Kattegat und die westliche Ostsee (Kieler-, Mecklenburger Bucht, Schärengürtel vor Stockholm). Diese äußersten Vorkommen fallen fast ausnahmslos in die Monate Juni bis Januar; es sind wohl meist ausgelaichte Tiere (bis $19 \mathrm{~cm}$ lang) und Jungfische $(7-10 \mathrm{~cm})$, die sich so weit verirren.

Im Herbst verläßt die Sardelle wieder die Nordsee, wandert diesmal aber durch den nördlichen Teil des Kanals, so daß sie nun von November bis Dezember regelmäßig und in größerer Häufigkeit längs der englischen Küste von Dover, Devon und Cornwall erscheint. Cunningham $(1895,1896)$ vermutet ihre Winterquartiere in und vor dem Westausgang des Kanals, wo Sardellen in den Wintermonaten all jährlich ziemlich häufig anzutreffen sind, bei Plymouth gelegentlich bis in den April.

2. Seit 1930 eingetretene Veränderungen

So lagen die Verhältnisse im nördlichen Verbreitungsgebiet bis etwa zum Jahre 1930. Um diese Zeit setzt in der südlichen Nordsee ein unvermittelter Anstieg des Sardellenbestandes ein, der auch noch in den folgenden Jahren bemerkbar bleibt. In der Zuider Zee steigt der Fangertrag des Jahres 1930

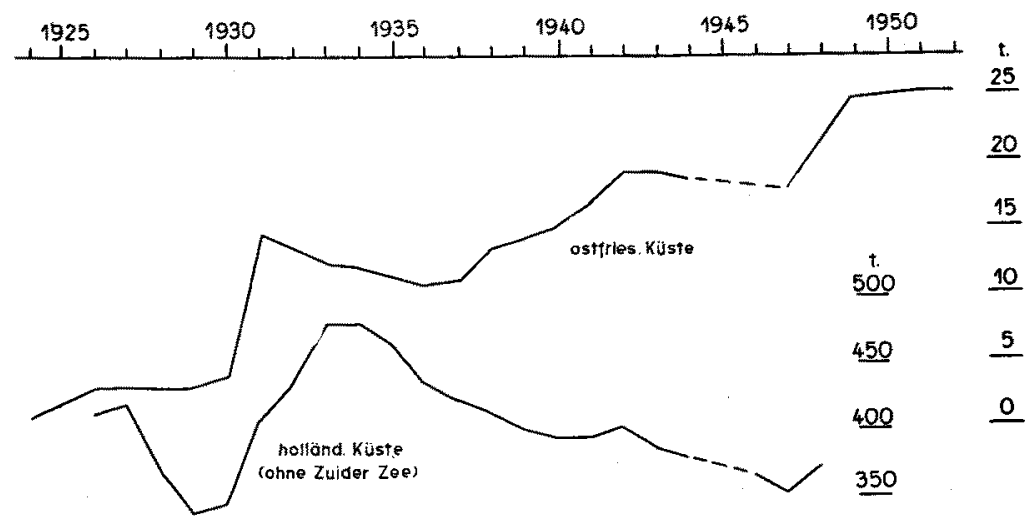

Abb. 1. Langjähriges Mittel der Sardellen-Anlandungen an der ostfriesischen (ab 1920) und holländischen (ohne Zuider Zee; ab 1922) Küste

auf fast das 7 fache des Durchschnittsertrages der Vorjahre (1922-1929) bzw. auf das $3^{1 / 2}$ fache des langjährigen Durchschnitts (1840-1931) an, in welchem Zeitraum er nur einmal, durch den Rekordfang des Jahres 1890, übertroffen wird. Ebenso liegen die Erträge aus dem holländischen Küstengebiet unter Ausschluß der Zuider Zee (Abb. 1) von 1930-1932 über der Norm und bewirken ein Ansteigen des langjährigen Mittels (1922-1929). Aber auch aus dem ostfriesischen Fanggebiet (Dollart, Ems-Mündung, Norddeich, Jadebusen) werden für die Jahre 1930-1934 und 1938 außergewöhnlich reiche Fänge gemeldet (P. F. Meyer 1930, 1932; Ehrenbaum 1932; Hass 1938; Hagmeier 1939). Besonders deutlich zeigen dies die Anlandungen der ostfriesischen Küstenfischerei (Abb. 1), die gegenüber dem langjährigen Durchschnitt 1931 
sprungartig auf das über $3^{1 / 2}$ fache des bisherigen Wertes (1920-1930) emporschnellen ${ }^{1}$.

In den 30er Jahren erscheinen erstmalig Sardellen auch vor der ElbeMündung und bei Helgoland, von wo sie bisher nur als äußerst seltene Gäste bekannt waren, in Zusammenhang mit einem verstärkten Zustrom salzreichen Wassers, der sich unter anderem durch das Auftreten außergewöhnlich großer Mengen von Sepia-Schulpen im Angespül der Insel bemerkbar machte (EHRENBaUM 1932). Aber noch über die Nordsee hinaus wirkt sich die Zunahme des Sardellen-Bestandes aus: im November-Dezember 1933 tauchen größere Sardellenschwärme, untermischt mit Sprott und Hering, in der westlichen Ostsee auf und ermöglichen hier Fangausbeuten bis zu 50-100 kg je Boot und Tag (Meyer 1933; Fischer 1935); vereinzelt gelangen Sardellen sogar bis zur Samland-Küste Ostpreußens (BAHR 1933).

Auf den plötzlichen Anstieg der Erträge im holländisch-ostfriesischen Fanggebiet anfangs der 1930er Jahre folgt zunächst ein Absinken unter das langjährige Mittel. Während dies aber für die holländische Küste beinahe stetig bis 1947 verläuft und erst der übernormale Fang des Jahres 1948 der Kurve wieder steigende Tendenz gibt (Abb. 1), steigt die Kurve für das ostfriesische Gebiet bereits anfangs der 1940er Jahre erneut zu einem zweiten Gipfel im Jahre 1942 an, der übrigens auch für das holländische Gebiet schwach angedeutet ist. Von 1943 bis 1947 ändert sich das langjährige Mittel nur wenig - der Verlauf der Kurve ist für diesen Zeitraum wegen des Fehlens von Angaben aus den Kriegs- und Nachkriegsjahren unsicher -, bis sich 1948 die Kurve in steilem Anstieg zur jetzigen Höhe erhebt.

Aus dem Vergleich der langjährigen Mittel der Fangerträge beider Gebiete ergibt sich: Die zu Beginn der 1930er Jahre erfolgte 1. Welle vermehrter Einwanderung von Sardellen bewirkt im holländischen Raum nur eine vorübergehende, schwache Erhöhung des langjährigen Mittels, das danach wieder auf den Ausgangswert, ja sogar etwas darunter zurücksinkt zugunsten eines um so stärkeren Anstieges im ostfriesischen Gebiet. Um 1940-1942 muß eine 2. Welle der Einwanderung stattgefunden haben, die sich praktisch aber nur an der ostfriesischen Küste auswirkte. Eine 3. große Einwanderungswelle ist dann 1949-1950 erfolgt, wiederum nur im ostfriesischen Gebiet zu einer merklichen Erhöhung des langjährigen Fang-Durchschnitts führend.

\section{Rassen-Zugehörigkeit}

Fage, Lo Giudice und Hokk unterscheiden bei der Sardelle zwei gut voneinander getrennte "Rassen“: eine mediterrane Rasse, zu der die Sardelle des Mittelmeeres und des Schwarzen Meeres gehört, und eine at lantische Rasse, die Populationen des atlantischen Küstengebietes von Südwest-Europa und der Zuider Zee umfassend. Fuknestin \& Coupe glauben, noch eine dritte, nordwest-afrikanische Gruppe anschließen zu können.

Die heute in der Deutschen Bucht laichende Sardelle entspricht nach der absoluten Länge der geschlechtsreifen Tiere, nach der relativen Kopflänge, der größeren Zahl von Strahlen in der Dorsale und der Wirbel weitgehend der Zuider Zee-Population.

1) Für die freundliche Vermittlung der Angaben über die Fangerträge der ostfriesischen Sardellen-Fischerei sei auch an dieser Stelle Herrn Ob.Fischercirat Dr. Heidrich (FischereiAmt Hamburg-Altona) und Herrn Dr. Reinke (Landesfischereiverband Weser-Ems in OIdenburg) herzlich gedankt. 
Die Abweichungen in der Zahl der Dorsal-Strahlen beruhen darauf, daß FAGE die vordersten, durch Haut verdeckten Strahlen unbeachtet ließ, die bei unserem Material zwar auch durch Haut eng mit dem dritten Strahl verbunden, aber immer deutlich erkennbar waren und daher mitgezählt wurden. Die Länge des ersten Strahles betrug etwa $1 / 6-1 / 5$, die des zweiten $1 / 2$ derjenigen des dritten Strahles.

\begin{tabular}{|c|c|c|c|c|}
\hline & Mittelmeer & $\begin{array}{l}\text { Zuider } \\
\left.\text { Zee } e^{x^{*}}\right)\end{array}$ & \multicolumn{2}{|c|}{ Deutsche Bucht } \\
\hline $\begin{array}{l}\text { Gesamtlänge } \\
\text { d. geschlechts- } \\
\text { reifen Tiere } \\
\text { in } \mathrm{cm}\end{array}$ & $\begin{array}{l}\text { 12-13 erstmalig } \\
\text { laichreif } \\
14-17 \text { zum 2. Mal } \\
\text { laichend }\end{array}$ & $\frac{11-14.5-18}{17-21}$ & $12.8-14.8-18.4$ & $17.5-18.5-19.6$ \\
\hline $\begin{array}{l}\text { Kopflänge } \\
\text { in } \% \text { der } \\
\text { Gesamtlänge }\end{array}$ & $21.0-22.4$ & $18.9-21.2$ & $19.0-21.0-22.9$ & $18.4-19,6-20.6$ \\
\hline $\begin{array}{l}\text { Strahlen in } \\
\text { der Dorsale }\end{array}$ & $12-13-13.7-15$ & $13-14.1-15$ & $15-16.1-17$ & 16 \\
\hline Wahl der & $44-44.88-45.96-48$ & $45-46.5-48$ & $\begin{array}{c}45-4 \\
\sigma= \\
\mathrm{m}= \\
\mathrm{n}\end{array}$ & $\begin{array}{l}1-48 \\
0.75 \\
0.13\end{array}$ \\
\hline
\end{tabular}

*) Nach Fage und Lo Giudice

*) Nach FAGE, Hozk und Redeke

4. Laichverhältnisse vor 1948

Bis zum Jahre 1929 wurde Sardellen-Brut innerhalb der Nordsee nur im holländischen und ostfriesischen Küstengebiet beobachtet, dagegen nicht mehr bei Helgoland, wo nur selten einmal vereinzelte Jungfische auftraten, ebensowenig vor der nordfriesischen und jütischen Küste. Ihre Häufigkeit blieb, wenn man die Zuider Zee als den einzigen bedeutsamen Laichplatz der Sardelle in der Nordsee außer Betracht läßt - hier erreichten die Eier eine Häufigkeit von durchschnittlich 200, maximal 2268 je $\mathrm{m}^{2}-$, ganz unbeachtlich und überstieg nicht den Wert von $2 / \mathrm{m}^{2}$. Gewöhnlich war die Brutdichte viel geringer, meist traten die Eier oder Larven nur in den reicheren Horizontalfängen in Erscheinung. Aber auch die Verbreitung der Brut war sehr beschränkt, so daß während der verschiedenen Fahrten Sardellen-Brut immer nur auf den wenigen, unmittelbar vor den Inseln oder zwischen diesen und der. Festlandsküste gelegenen Stationen überhaupt nachzuweisen war. Eine einzige Ausnahme machte die Beobachtung Ehrenbaums (1892a), der am 7. Juli $18917 \mathrm{sm}$ nordwestlich Norderney in drei Brutnetzfängen von insgesamt 20-30 min Schleppdauer rund 16000 Sardellen-Eier fing (das sind schätzungsweise 500 je $\mathrm{m}^{2}$ ). Auf Grund dieser Beobachtung glaubte EHreNBAUM annehmen zu dürfen, daß die eigentlichen Laichplätze der Sardelle auf offener See lägen, wenn auch nicht weit ab von der Küste, und nicht, wie die holländischen Forscher bisher annahmen, in der Zuider Zee. Er mußte aber später (1908) diese Ansicht widerrufen, da in den folgenden Jahren trotz intensiver Nachsuche im ostfriesischen Seegebiet Sardellen-Brut nur ganz vereinzelt und in geringer Häufigkeit wiedergefunden werden konnte (BoEKE 1906; Ehrenbaum 1892b, 1908; Heincke \& Ehrenbaum 1900; Redeke 1913/19, 1916; TESCH 1909, 1913/19). 
1930, als sich auch die Beobachtungen erwachsener Sardellen mehrten, erfolgte eine deutliche Zunahme des Laichens, so daß von da ab auf allen während der Laichzeit der Sardelle in der südlichen Nordsee unternommenen Fahrten Eier und Larven angetroffen wurden. Ihre Häufigkeit stieg bis auf $24 / \mathrm{m}^{2}$, gleichzeitig erweiterte sich ihre Verbreitung teils nach See $z u$, teils über die Elbe-Mündung und Helgoland hinaus bis vor das Lister Tief und den Limf jord; im Juni 1930 wurde Sardellen-Brut auf 15\% aller innerhalb der Deutschen Bucht gelegenen Stationen beobachtet. Ab 1938 traten erstmalig Sardellen-Eier auch im Kattegatt und sogar in der westlichen Ostsee auf (Aurich 1940; Ehrenbaum 1932; Hagmeier 1939; Heegaard 1947; KändLer \& Wattenberg 1940, Meyer 1930, 1932).

\section{Laichverhältnisse seit 1948}

Eine zweite, wesentlich bedeutsamere Steigerung des Sardellen-Laichens konnte nach dem letzten Kriege, als die Fischbrut-Untersuchungen in der Deutschen Bucht wieder aufgenommen wurden, festgestellt werden. Über die Verhältnisse im Jahre 1947 läßt sich nichts aussagen, da die Untersuchungen erst im August begannen und auf das Lister Wattenmeer beschränkt blieben. 1948 aber tritt Sardellen-Brut von Mitte Juni an bis Ende Juli regelmäßig, wenn auch in mäßiger Häufigkeit, im Plankton bei List auf. Während einer Fahrt vom 29.-31. Juli in das nordfriesische Seegebiet zwischen Horns Riff und Helgoland werden zwar keine Eier gefangen - es wurde nur mit dem Knüppelnetz gefischt, von dẹm die Sardellen-Eier nicht vollständig zurückgehalten werden -, dagegen sind Larven über das ganze Gebiet in großer Häufigkeit verbreitet. Sie werden auch noch während der folgenden Fahrt vom 1.-9. Oktober im gleichen Gebiet auf der Hälfte aller Stationen angetroffen, besonders auf den küstennäheren Stationen von der Eider bis ins Lister Tief, fehlen aber bei Helgoland und im Graa Dyb; meist sind es ältere, bereits in Schuppenbildung begriffene Stadien von $30-41 \mathrm{~mm}$ Länge neben wenigen jüngeren Larven von $17-27 \mathrm{~mm}$.

1949 erscheinen die ersten Sardellen-Eier bei List am 27. Mai. Anfang Juli sind sie hier am häufigsten und verschwinden Ende dieses Monats wieder aus dem Plankton. Larven können noch bis in die zweite Augusthälfte beobachtet werden. Die Eier sind bis in die inneren Gebiete des Wattenmeeres verbreitet, nehmen aber gegen das Land hin an Häufigkeit ab. Wahrscheinlich werden sie nur durch die Gezeitenströmung hierher verdriftet, die Maxima ihrer Verbreitung, also die Laichzentren, treffen wir erst auf offener See an.

Vom 20. Juni-5. Juli wurde zur besonderen Untersuchung der Verbreitung der Sardellenbrut eine Fahrt längs der deutschen Nordsee-Küste unternommen, wobei die Stationen mehr in Küstennähe gelegt wurden in der, wie sich zeigen sollte, falschen Annahme, daß die Sardelle, entsprechend den früheren Verhältnissen an der holländischen und ostfriesischen Küste, zum Laichen die Küstengewässer bevorzugt aufsuche. Das Ergebnis dieser Fahrt ist in Abb. 2 dargestellt.

Auf beinahe allen Stationen (93\%) wird Sardellenbrut in nicht erwarteter Häufigkeit angetroffen. Die Höchstwerte betragen 3885 Eier, $594 \mathrm{Larven} / \mathrm{m}^{2}$, und der Durchschnitt für die Stationen mit 100 und mehr Eiern je $\mathrm{m}^{2}$ liegt bei $660 / \mathrm{m}^{2}$. Das sind Brutdichten, die diejenigen aller anderen Fischarten mit pelagischen Eiern in der südlirhen Nordsee zum Teil weit übertreffen: 


\begin{tabular}{llll}
\multicolumn{4}{c}{ Maximale Brutdichten in der südlichen Nordsee } \\
\hline Sprott & 400 Eier $/ \mathrm{m}^{2}$ & (im Skagerrak bis & 2000) \\
Kabeljau & 377 & Kliesche & 1300 Eier $/ \mathrm{m}^{2}$ \\
Wittling & $621 \quad$ & Scholle & $576 \% "$
\end{tabular}

Aber auch die früher in der Zuider Zee festgestellten Brutdichten werden 1949 noch erheblich übertroffen: dort lagen die Höchstwerte in den Jahren 1906-1912 meist zwischen 50 und $300 \mathrm{Eier} / \mathrm{m}^{2}$, nur selten wurden höhere Werte bis 681 und nur einmal der extreme Wert von 2268 erreicht (REDekF 1913/16); als Durchschnittswert ergibt sich hieraus für die Zuider Zee etwa $200 \mathrm{Eier} / \mathrm{m}^{2}$.

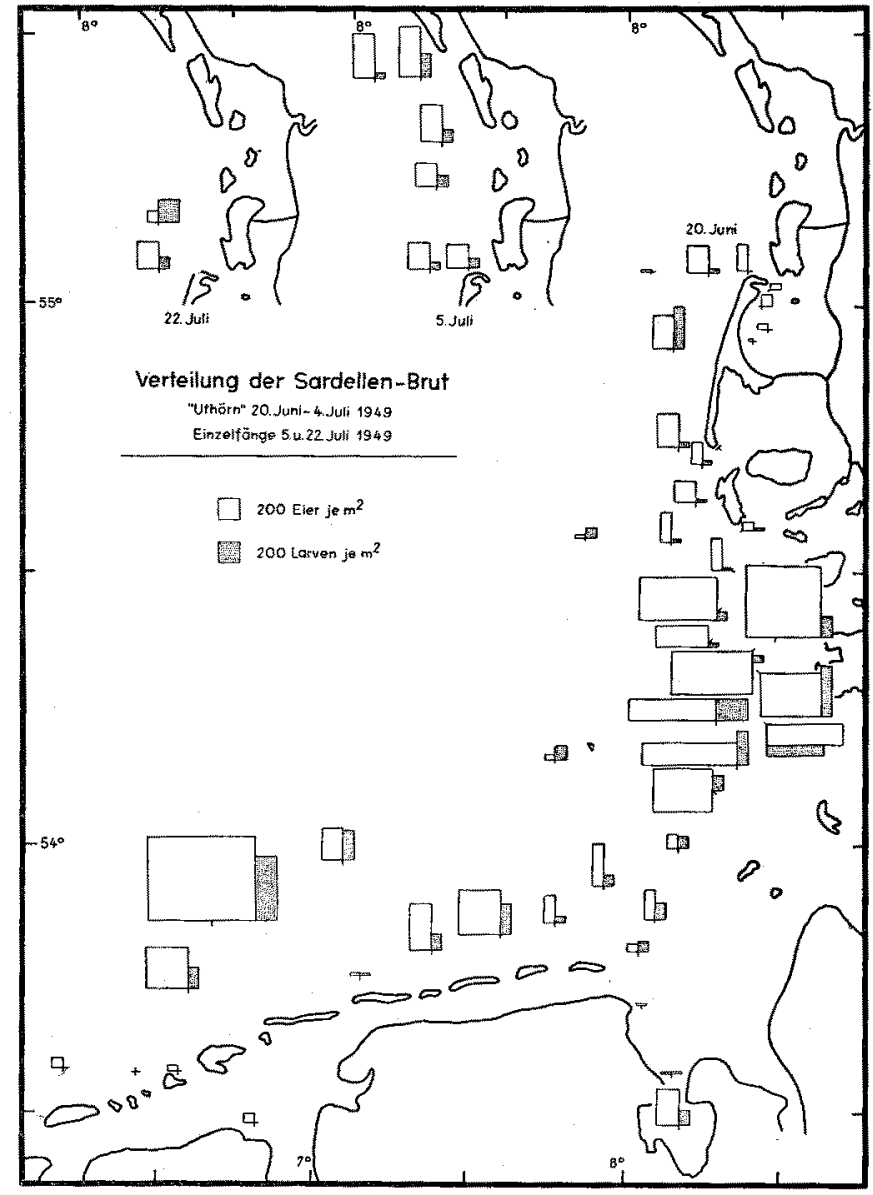

Abb. 2. Verteilung der Sardellen-Brut im Juni und Juli 1949

Trotz der geringen Dichte des Stationsnetzes dieser Fahrt ist eine Aufgliederung des gesamten Brutvorkommens in einzelne Laichplätze erkennbar, die auch in den folgenden Jahren immer wieder festzustellen sind:

1. Ein umfangreicher Laichplatz zeichnet sich vor der ostfriesischen Küste $a b$, wo nördlich Borkum der größte Fang, 3885 Eier und 594 Larven unter dem Quadratmeter, gemacht wurde. Die mittlere Laichdichte (Durchschnitt aller Stationen mit mehr als $100 \mathrm{Eier} / \mathrm{m}^{2}$ ) beträgt für dieses Gebiet $781 \mathrm{Eier} / \mathrm{m}^{2}$. 
2. Von ihm wird durch das Weser-Elbe-Wasser ein zweiter Laichplatz im Gebiet Eider - Hever abgetrennt, mit durchschnittlich 1065, maximal $2367 \mathrm{Eier} / \mathrm{m}^{2}$.

3. Von geringerer Bedeutung und in diesem Jahre nur undeutlich in Höhe Amrum von dem vorhergehenden abgesetzt, folgt ein dritter Laichplatz im Gebiet querab Sylt-Lister Tief-Graa Dyb. Seine mittlere Laichdichte beträgt im Juni $249 \mathrm{Eier} / \mathrm{m}^{2}$, erreicht am 5. Juli ihr Maximum mit 317 und sinkt dann wieder ab auf 152 am 22. Juli. Demgegenüber wächst die durchschnittliche Häufigkeit der Larven fortlaufend an, wahrscheinlid auch durch Zudrift aus den südlicheren Gebieten, von $39 / \mathrm{m}^{2}$ im Juni auf 52 am 5 . Juli und 134 am 22. Juli.

Während einer späteren Fahrt vom 7.-11. September, die anderen Aufgaben diente und nur das nordfriesische Gebiet berührte, werden SardellenEier nicht mehr beobachtet; dagegen kommen Larven auf $78 \%$ aller Stationen vor, am häufigsten vor dem Lister Tief, fehlen aber bei Helgoland. Ihre Dichte hat gegenüber den Sommermonaten erheblich abgenommen:

$$
\begin{array}{lllll}
\text { Zahl der Larven je } & \text { 3.-5. Juli } & \text { 22. Juli } & \text { 15.-20. August } & \text { 7.-11.September } \\
\text { Knüppelnetz-Fang } & 42-148 & 4867 & 77-101 & 12-93 \\
\text { im Lister Tief } & & & &
\end{array}
$$

Am 19. September und später werden keine Larven mehr angetroffen.

1950 werden während einer Fahrt in der ersten Maihälfte im nordfriesischen Gebiet noch keine Sardellen-Eier beobachtet. Sie erscheinen bei List erst am 22. Mai und erreichen wenige Tage später hier eine Häufigkeit von $54 / \mathrm{m}^{2}$. Auf den vom 1.-8. Juni und 7.-14. Juli folgenden, weiter ausgedehnten Fahrten sollten möglichst die Grenzen des Laichvorkommens nach Westen hin und nach der offenen See zu festgestellt werden. Das Ergebnis der beiden Fahrten ist in den Abb. 3 und 4 in Form von Isoplankten dargestellt.

Anfang Juni sehen wir das Laichen in der ganzen Deutschen Bucht in vollem Gange. Auf dem weitaus größeren Teil der Stationen - im Juni auf $78 \%$, im Juli auf $89 \%$ - wird Sardellen-Brut beobachtet, nur die am weitesten in See gelegenen Stationen sowie wenige im engeren Küstenbereich bleiben negativ. Wie im vorhergehenden Jahre lassen sich drei voneinander abgesetzte Laichplätze erkennen, von denen die beiden südlichen eine Tendenz $z u$ weiterer Aufteilung zeigen. Diese wird bedingt durch das sich keilförmig einschiebende Mischwasser vor der Ems- ("ostfriesisches Mischwasser") und Elbe-Mündung (vgl, hierzu die in Abb. 7 eingetragene Verteilung des Salzgehalts). Entsprechende Verhältnisse konnten schon früher für die Verteilung der Sprott-Brut festgestellt werden (AURICH 1940, S. 187). Vor dem Lister Tief und im westlichen Teil des friesischen Laichplatzes hält sich das Laichen während beider Monate etwa auf gleicher Höhe; dagegen hat es sich auf dem Helgoland-Hever-Laichplatz erheblich zugunsten des westlich angrenzenden Gebietes abgeschwächt, von 900 auf $300 \mathrm{bzw}$. 100 Eier $/ \mathrm{m}^{2}$. Im Juni nämlich wird dieses Gebiet durch einen von Nordwesten gegen die Weser-Mündung vorgeschobenen Keil kälteren Wassers (Oberflächen-Temperatur unter $12^{\circ}$ ) noch für das Laichen der Sardelle blockiert, während im Westteil des ostfriesischen Laichplatzes die Temperaturen 12-13,50, auf dem HelgolandHever-Laichplatz sogar $14-16,5^{0}$ betragen. Im Juli sind dann diese Unterschiede ausgeglichen: 


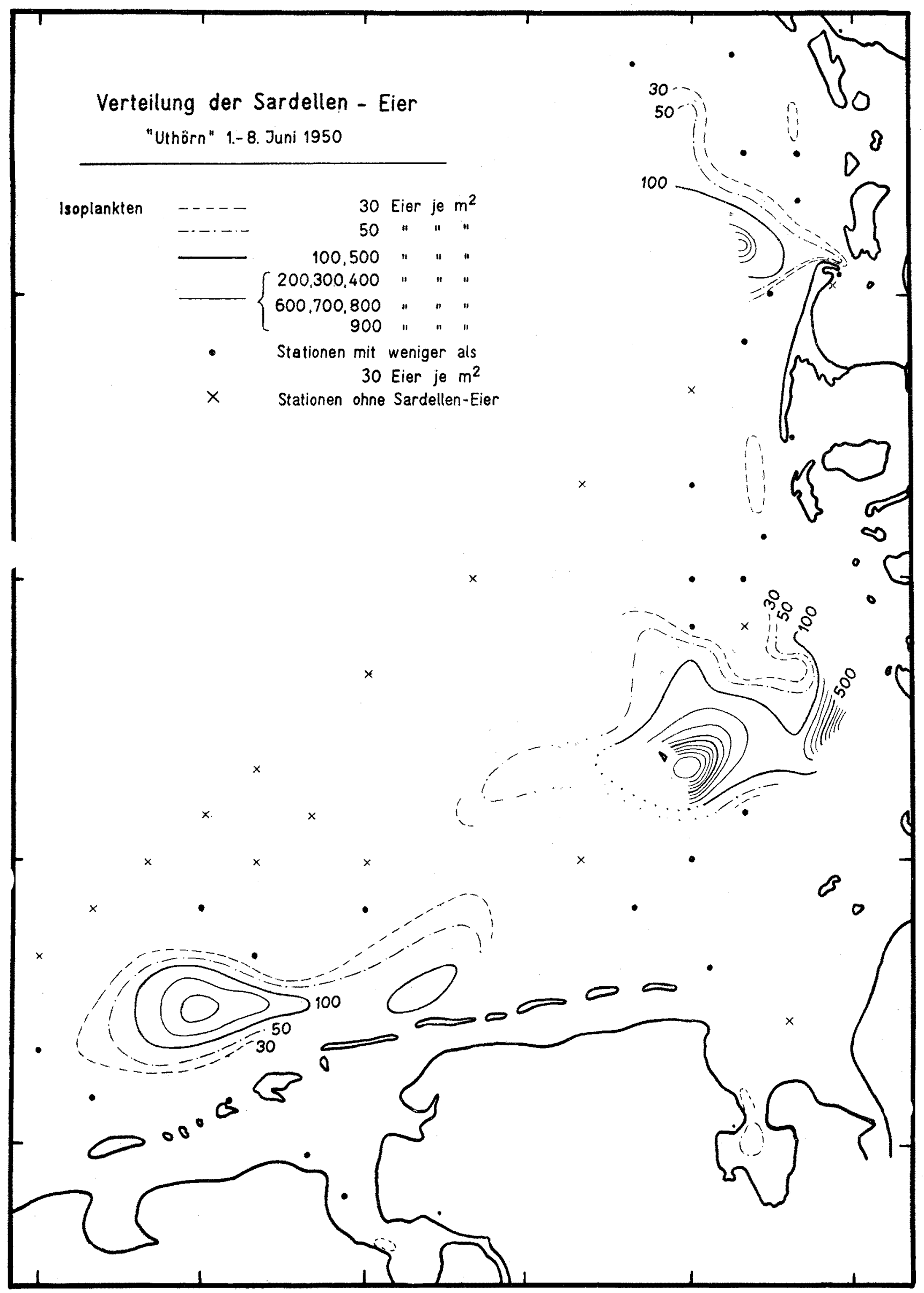

Abb, 3. Verteilung der Sardellen-Eier im Juni 1950 


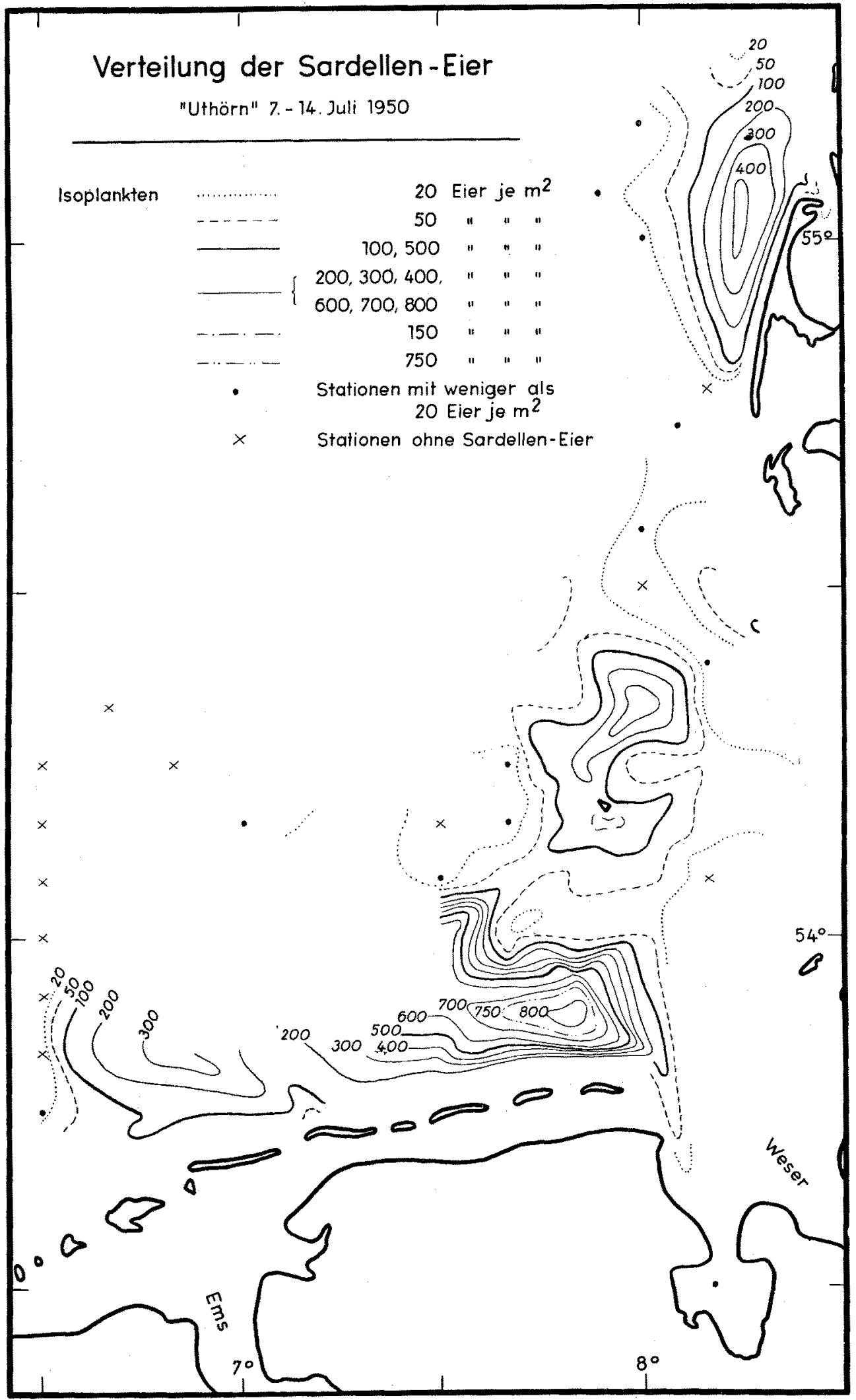

Abb. 4. Verteilung der Sardellen-Eier im Juli 1950 


$\begin{array}{ll}\text { Westteil des friesischen Laichplatzes } & 17-18^{0} \\ \text { Ostteil des friesischen Laichplatzes } & 16,5-17,5^{\circ} \\ \text { Helgoland-Hever-Laichplatz } & 17-18,5^{\circ},\end{array}$

so daß die Laichschwärme nun auch in das Gebiet querab Langeoog-Wangerooge eindringen können (? Rückwanderung aus dem Raum HelgolandHever).

Auf dem Lister-Tief-Laichplatz konnten die Laichverhältnisse über einen größeren Zeitraum verfolgt werden:

\begin{tabular}{cccc} 
& $\begin{array}{c}\text { Oberfächen- } \\
\text { Temperatur }\end{array}$ & $\begin{array}{c}\text { Eier } \\
\text { je } \mathrm{m}^{2} \\
\text { (sofern nicht anders vermerkt) }\end{array}$ & $\begin{array}{c}\text { Larven } \\
\text { je } \mathrm{m}^{2}\end{array}$ \\
\hline 3.-11. Mai & $9,9-12,1$ & 0 & 0 \\
22. Mai & 13,7 & 1 (qualitativer Fang) & 0 \\
25. Mai & 14,0 & 54 & 0 \\
1. Juni & 14,0 & 24 & 0 \\
8. Juni & 17,3 & 153 & 9 \\
14. Juni & 16,3 & 165 & 15 \\
27. Juni & 17,8 & 414 & 9 \\
7. Juli & 16,5 & 369 & 114 \\
14. Juli & 19,8 & 429 & 222 \\
20. Juli & 19,3 & 366 & 99 \\
31. Juli & 18,3 & 0 & 0 \\
2. August & 18,4 & 12 & 0 \\
14. August & 19,1 & 0 (Knüppel-Netz) & 9 (Knüppel-Netz) \\
22. August & 18,9 & 0 (Knüppel-Netz) & 3 (Knüppel-Netz)
\end{tabular}

Das Laichen beginnt demnach in den letzten Mai-Tagen, sobald sich das Wasser auf $13^{\circ}$ und mehr erwärmt hat, nimmt allmählich an Intensität $\mathrm{zu}$ bis zur Hochzeit (über $300 \mathrm{Eier} / \mathrm{m}^{2}$ ), die von Ende Juni bis über Mitte Juli anhält, und klingt dann sehr rasch ab. Die Gesamtdauer des Laichens beträgt reichlich 10 Wochen.

Die Verteilung der Sardellen-Brut während der beiden Sommerfahrten 1951 entspricht in großen Zügen der der Vorjahre. Im Juni (Abb.5) beginnt das Laichvorkommen im Westen in Höhe Ameland-Schiermonnikoog und erstreckt sich die ostfriesischen Inseln entlang mindestens bis Norderney, wo die meisten Eier $\left(357 / \mathrm{m}^{2}\right)$ angetroffen werden. Durch eine schmale, ostwärts gerichtete Zunge salzreicheren und kälteren Wassers - die OberflächenTemperatur beträgt innerhalb dieser Zunge nur 12,9-14 $0^{0}$, nördlich und südlich davon jedoch $14,0-15,6^{\circ}$ - wird das ostfriesische Laichvorkommen auf einen gegenüber den Vorjahren schmäleren Streifen dicht vor den Inseln eingeengt. Im Ostteil dieses Gebietes wurde durch die erwähnte WestwasserZunge jedoch ein Teilschwarm von der Küste abgedrängt und laicht nun isoliert in der offenen See. Eine schmale, von der Jade-Wêser-Mündung nach Nordwesten verlaufende Zone mit Temperaturen unter $14^{\circ}$, in der höchstens dorthin verdriftete Larven beobachtet werden, bildet die Grenze zum Helgoland-Hever-Laichplatz, auf dem die Brutdichte bis $123 \mathrm{Eier} / \mathrm{m}^{2}$ beträgt. Weiter nach Norden folgt dann ein völlig brutleeres Gebiet bis querab Mitte Sylt, durch das der Laichplatz im Lister Tief-Graa Dyb abgetrennt wird. Die Laichintensität ist hier mit maximal $93 \mathrm{Eier} / \mathrm{m}^{2}$ wieder am schwächsten.

Bis zur folgenden Fahrt vom 10.-13. Juli (Abb.6) hat sich die Brutverteilung nicht wesentlich geändert. Auf dem friesischen Laichplatz ist zwar 
die Brutdichte noch etwas angestiegen, der hohe Anteil an Larven deutet aber darauf hin, daß der Höhepunkt des Laichens hier bereits überschritten ist (200-460 Eier, 100-246 Larven $/ \mathrm{m}^{2}$ ). Dagegen befinden sich bei HelgolandHever die Eier noch weitaus in der Uberzahl (100-534 Eier gegenüber höchstens $57 \mathrm{Larven} / \mathrm{m}^{2}$ ) und sind hier auch noch am 6 . August reichlich vertreten (246 Eier, 12 Larven $/ \mathrm{m}^{2}$ ). Nach Mitte August kommen im nordfriesischen Gebiet nur noch Larven vor, fehlen aber in den im September und Oktober gemachten Fängen.

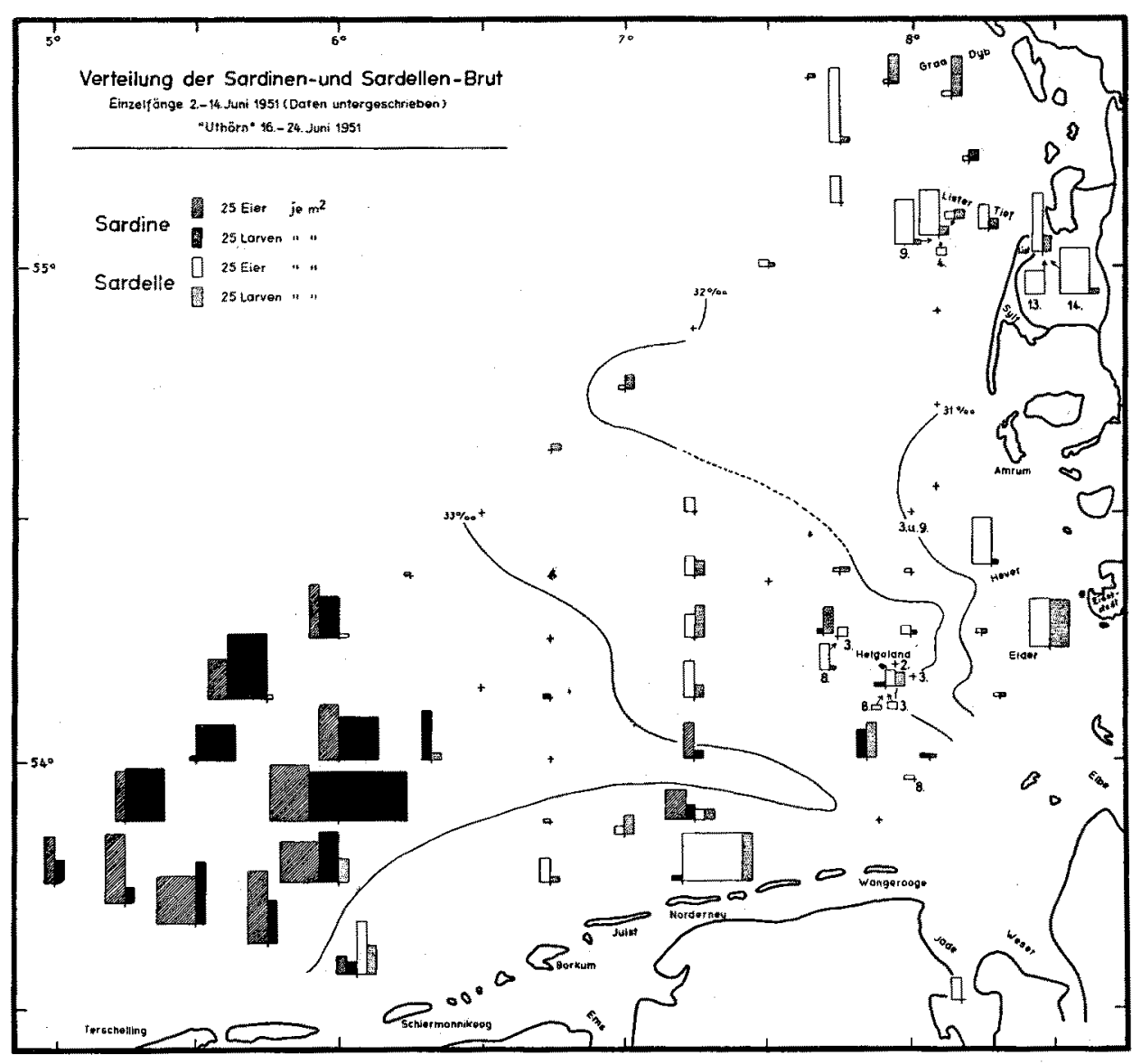

Abb. 5. Verteilung der Sardinen- und Sardellen-Brut im Juni 1951

Bei List treten die ersten Eier am 24. Mai auf, als die Temperatur erstmals $13^{0}$ überstiegen hat, sie verschwinden dann aber infolge eines Rückschlages der Temperatur unter $11^{0}$ kurzfristig nochmals aus dem Plankton und erscheinen erst am 30. Mai nach erneuter Erwärmung wieder:

Die obenstehende Übersicht über die auf dem Lister-Tief-Laichplatz beobachteten Brutdichten zeigt ferner, daß das Laichen 1951 schwächer bleibt als in den Vorjahren und außerdem früher zu Ende geht.

Auch 1952 verschwinden bei List die Eier, die am 28. Mai erschienen waren, nachdem die Temperatur wenige Tage zuvor $13,5^{\circ}$ überschritten hatte, Ende des Monats nochmals, als sich das Wasser auf $12^{\circ}$ abkühlt, und treten erst vom 3. Juni ab nach erneutem Temperaturanstieg wieder regelmäßig in 
den Planktonfängen auf. Mitte Juni wird bereits eine Häufigkeit von 174 Eier $/ \mathrm{m}^{2}$ erreicht.

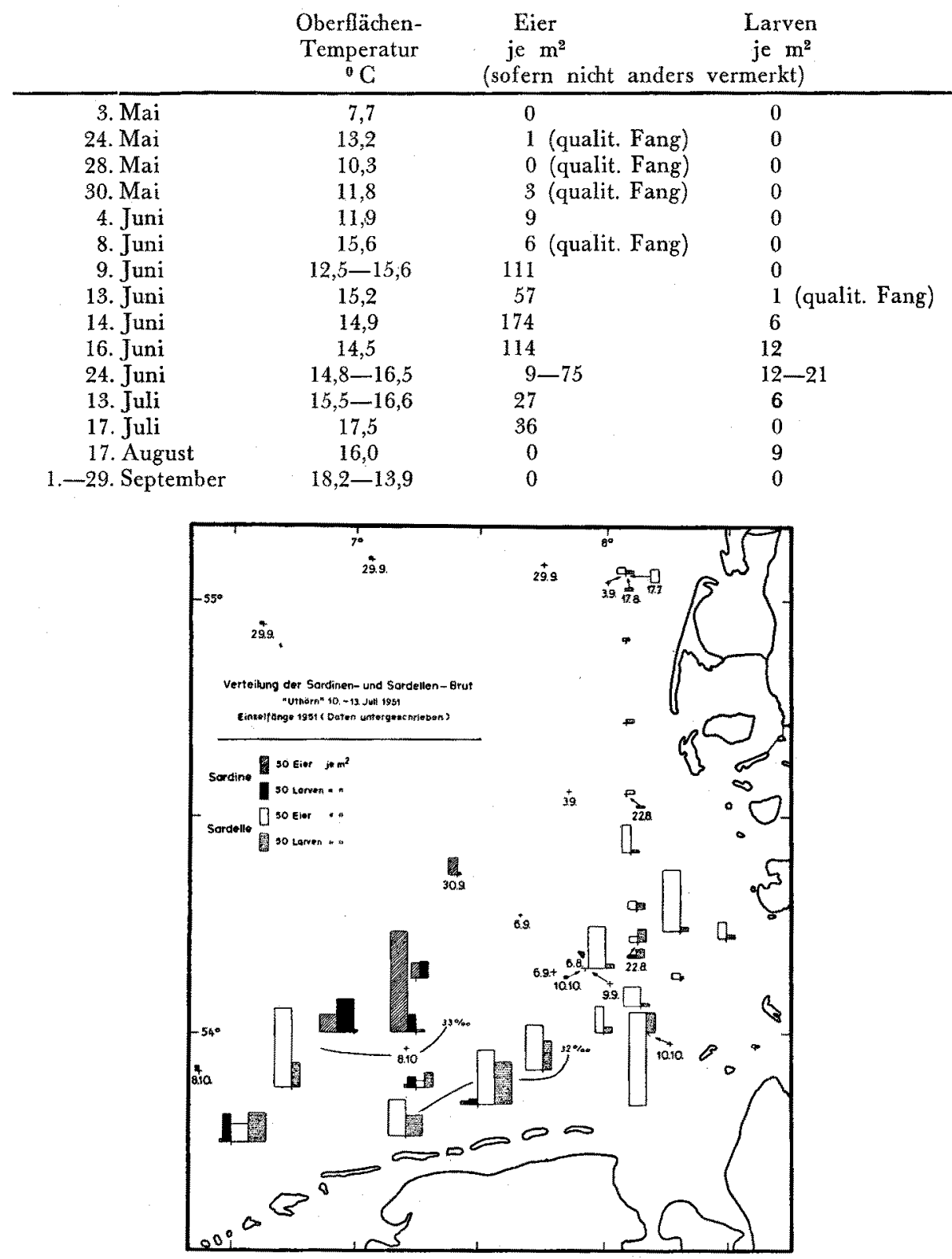

Abb.6. Verteilung der Sardinen- und Sardellen-Brut im Juli (Stationen obne Angabe des Datums) und August-Oktober (Stationen mit beigeschriebenem Datum) 1951

Ebenso weist auch das während der Juli-Fahrt gewonnene Bild der Brutverteilung die gleichen Züge wie in den Vorjahren auf: Innerhalb des friesischen Laichgebietes sind wieder zwei durch das vor der Ems-Mündung liegende "ostfriesische Mischwasser" getrennte Kerngebiete zu erkennen, das eine nordwestlich Schiermonnikoog mit maximal 174 Eier, 75 Larven $/ \mathrm{m}^{2}$ und 
ein zweites querab Juist-Norderney mit maximal 456 Eier, $339 \mathrm{Larven} / \mathrm{m}^{2}$. Allgemein ist das Laichen hier schon weiter vorgeschritten, so daß die Larven auf der Mehrzahl aller Stationen $(62 \%)$ die Eier zahlenmäßig übertreffen. Auf dem Helgoland-Hever-Laichplatz zeigt sich das Laichen dagegen noch in vollem Gange. Die Eier herrschen auf $3 / 4$ der Stationen vor und erreichen eine Häufigkeit bis zu $891 / \mathrm{m}^{2}$. Ihre Verbreitung erstreckt sich in diesem Jahre besonders weit südwärts, bei Feuerschiff Elbe I und 8 sm westlich davon werden noch 213-723 Eier/ $\mathrm{m}^{2}$ beobachtet. Etwa in Höhe Amrum ist dann wieder eine deutliche Zone der Brutverarmung ausgeprägt, an die sich querab Mitte Sylt das Laichen vor dem Lister Tief anschließt. Die Eier sind überall in der Mehrzahl vorhanden, bis $390 / \mathrm{m}^{2}$, was darauf hinweist, daß hier das Laichen am spätesten begonnen hat.

Wie bei den früheren Fahrten bleibt das Laichen an das Küstenwasser gebunden, das sich in einem Streifen wechselnder Breite außerhalb der Inselketten entlangzieht und während der Juli-Fahrt durch eine starke Entwicklung von Noctiluca, in der inneren Deutschen Bucht außerdem durch eine Wucherung von Biddulphia sinensis und Coscinodiscus grani gekennzeichnet ist. Dagegen fehlt Sardellen-Brut völlig in dem seewärts anschließenden, bis $55^{\circ} \mathrm{N}, 4^{0} 30^{\prime} \mathrm{O}$ untersuchten Gebiet, das durch häufiges Auftreten von Aglantha

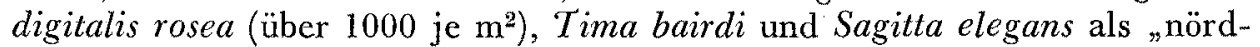
liches Wasser" charakterisiert ist.

6. Folgerungen aus der Verteilung der Sardellen-Brut

Zusammenfassend ergibt sich aus den Befunden über die Verteilung der Sardellen-Brut während der fünf Untersuchungsjahre:

1. Das Laichen der Sardelle findet nicht, wie nach früheren Verhältnissen im holländischen Seegebiet und den wenigen Beobachtungen von SardellenBrut an der deutschen Küste zu erwarten war, vornehmlich im engeren Küstenbereich statt, sondern die größten Brutdichten werden stets außerhalb der Inselketten auf offener See angetroffen.

2. Das gesamte Brutvorkommen in der südöstlichen Nordsee ist in drei mehr oder weniger scharf voneinander getrennte Laichplätze aufgegliedert, deren beide südlichere zu weiterer Aufspaltung neigen:

Friesischer Laichplatz: Von den westfriesischen Inseln bis vor die Weser-Elbe-Mündung reichend. Querab Borkum-Norderney verursacht das "ostfriesische Mischwasser" eine schwache Unterteilung des Gebietes in eine westliche und eine östliche Hälfte. Durch den nach Nordwesten vordringenden Keil des Weser-Elbe-Mischwassers wird es von dem

Helgoland-Hever-Laichplatz abgegrenzt, der das Gebiet zwischen Helgoland und der Halbinsel Eiderstedt umfaßt. Ein zweiter, nach Norden gerichteter Keil des Weser-Elbe-Wassers führt auch hier zu einer weiteren Unterteilung des Laichvorkommens.

Lister-Tief-Laichplatz. Von dem vorhergehenden wird er durch eine auffallend brutarme bis brutleere Zone abgetrennt, die etwa von Höhe Amrum bis Mitte Sylt reicht und auch durch eine besondere Verarmung der Bodenfauna gekennzeichnet ist. Flächenmäßig wie auch an Intensität des Laichens bleibt das Lister Laichgebiet hinter den beiden anderen zurück.

3. Das Gemeinsame und Kennzeichnende dieser drei Laichplätze, das sie die Sardelle bevorzugt aufsuchen läßt, beruht in ihren besonderen Temperaturverhältnissen während des späteren Frühjahres. Alle drei Gebiete 
liegen gerade in der Zone, in der sich das von Westen kommende, kühlere „atlantische" Wasser unter der Wirkung der Gezeiten mit dem sich im Frühjahr schneller erwärmenden Wasser des Wattenmeeres vermischt, wodurch auch seine Temperatur auf die von der Sardelle zum Laichen geforderte Höhe gehoben wird. Dank der größeren Tiefe der Mischzonen bleiben die Temperaturen dann verhältnismäßig konstant, während sie im Wattenmeer selbst zur gleichen Zeit noch erheblichen täglichen Schwankungen unterworfen sind.

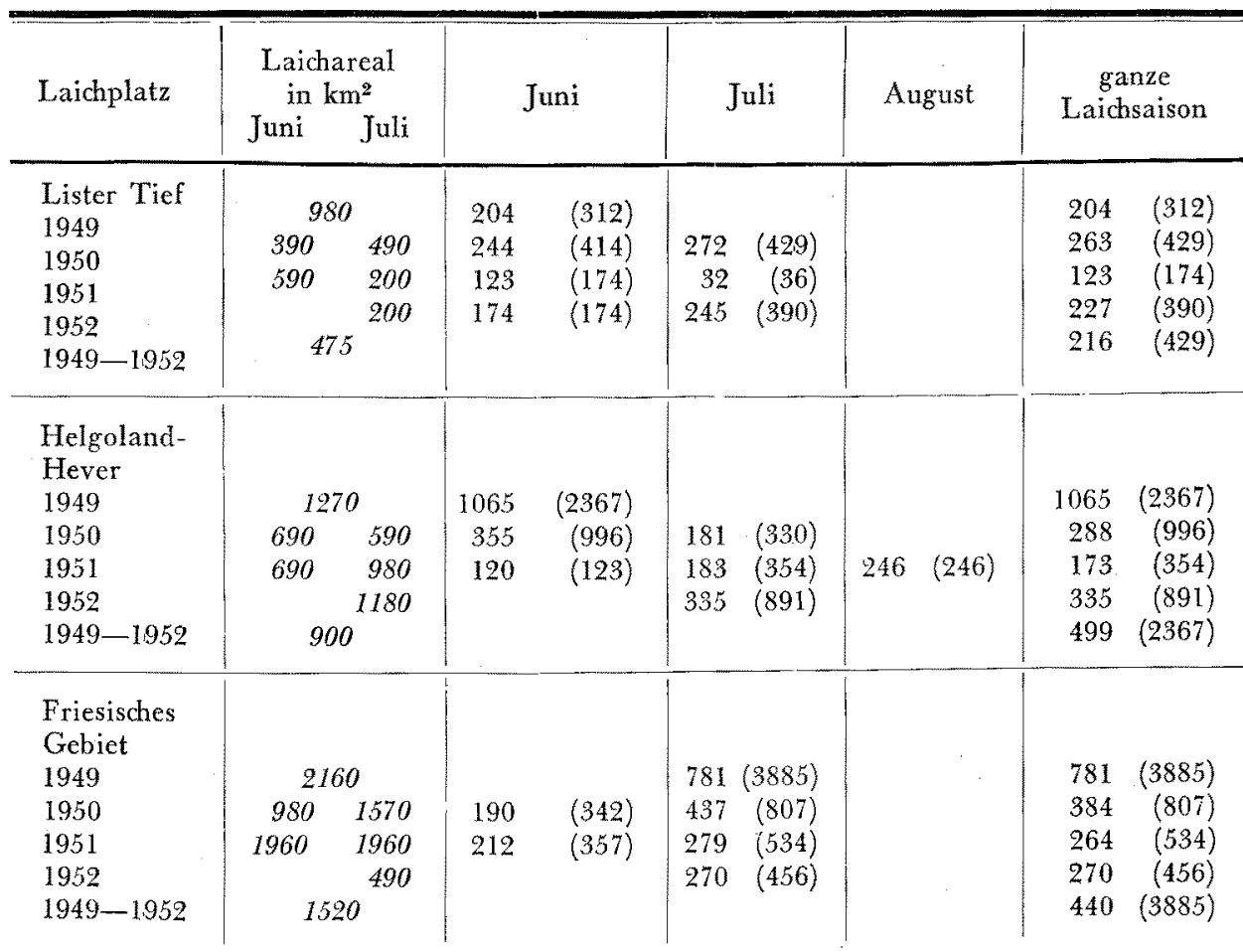

Durchschnittliche und maximale (in Klammern gesetzt) Häufigkeit der Eier je $\mathrm{m}^{2}$ innerhalb der Gebiete mit 100 und mehr Eiern je $\mathrm{m}^{2}$ sowie die Größe dieser Areale in $\mathrm{km}^{2}$.

4. Als untere Temperaturschwelle für das Laichen der Sardelle ergibt sich nach unseren Beobachtungen bei List etwa $13,0-13,5^{0} \mathrm{C}$. In allen Jahren erscheinen hier die ersten Eier, sobald dieser Wert überschritten wird, und verschwinden bei Temperaturrückschlägen wieder aus dem Plankton. FAGE (1911) stellte den gleichen Wert $\left(13^{0}\right)$ als untere Grenze für die Entwicklung der Gonaden bei der Sardelle fest.

Während der Hochzeit des Laichens, Mitte Juni bis Mitte Juli, beträgt die Temperatur in unserem Gebiet $15-20^{\circ}$ und hält sich in gleicher Höhe bis zum Ende des Laichens, das in die erste Augusthälfte fällt. Danach werden nur noch Larven gefangen, die sich im Oktober größtenteils in Metamorphose befinden (Schuppenbildung bei $32-34 \mathrm{~mm}$ Länge). In den während des November mit dem Knüppelnetz gemachten Fängen treten Larven nicht mehr auf; nur einmal (12. November 1952) wurde bei Helgoland noch ein Jungfisch $(50 \mathrm{~mm})$ erbeutet.

5. Im Gegensatz zu den Temperaturverhältnissen läßt sich keinerlei Beziehung zwischen Brutverteilung und Salzgehalt feststellen, vielmehr laicht die 
Sardelle sowohl im salzreichen „Westlichen Wasser" von über 33\% wie auch im brackigen Wasser der Buchten und Flußmündungen bis zu 17\% herab.

6. Eine Berechnung der absoluten Laichproduktion im Sinne von HENSEN und BuchanaN-Wollaston, nämlich durch Integration der zu mehreren Zeitpunkten innerhalb einer Laichperiode ermittelten Eimengen, scheitert leider daran, daß wir Brutverteilung und -häufigkeit in der Regel nur zweimal während einer Laichperiode bestimmen konnten. Dafür läßt sich ein relatives Maß für das Laichen auf den verschiedenen Plätzen und während der einzelnen Jahre aus der Laichintensität, gemessen als durchschnittliche Häufigkeit der Eier innerhalb der Gebiete mit über $100 / \mathrm{m}^{2}$, unter Berücksichtigung der Fläche dieser Gebiete ableiten (s. nebenstehende Tabelle).

Im allgemeinen ist die Intensität des Laichens in den beiden südlichen Gebieten stärker, im Durchschnitt der Jahre etwa doppelt so groß wie auf dem Lister Tief-Laichplatz. Noch größer sind die Unterschiede hinsichtlich der Laichareale, für die allerdings nur Annäherungswerte gegeben werden können, da die einzelnen Gebiete nicht auf allen Fahrten in ihrer gesamten Ausdehnung erfaßt wurden. Aus beiden Größen, gemittelt für die ganze Beobachtungszeit, ergibt sich dann folgendes Verhältnis der Laichbestände:

\begin{tabular}{lccc} 
Laichplatz & Laichintensität $\times$ Laichareal $=$ relative Bestandsgröße \\
\hline Lister Tief & 1 & 1 & 1 \\
Helgoland-Hever & 2 & 2 & 4 \\
Friesisches Gebiet & 2 & 3 & 6
\end{tabular}

Soweit diese Unterschiede nicht rein topographisch durch die Größe der Gebiete, in denen die Voraussetzungen für ein Laichen der Sardelle erfüllt werden, bedingt sind, muß man sie darauf zurückführen, daß die Masse der Schwärme auf dem ersten, ihren Laichanforderungen genügenden Platz - das ist jetzt nach dem Wegfall der Zuider Zee das Gebiet vor den friesischen Inseln - verbleibt und nur immer kleiner werdende Schwärme, die Küste entlang wandernd, bis in die nordöstlichen Gebiete gelangen. Darauf deutet auch der sich aus dem Verhältnis Eier/Larven ergebende spätere Beginn des Laichens im nordfriesischen Raume hin.

7. Das umfangreiche Laichen der Sardelle in der südöstlichen Nordsee findet seit mindestens 1948 statt und mag in diesem Jahre, nach der Häufigkeit älterer Larven im Ostteil der Deutschen Bucht während der Oktoberfahrt, von etwa gleicher Stärke gewesen sein wie das 1949 unmittelbar beobachtete Laichen mit den Rekordwerten von ein- bis fast viertausend Eiern je Quadratmeter. In den folgenden Jahren nimmt, wenigstens auf den beiden südlichen Laichplätzen, die Brutdichte wieder etwas ab, ohne jedoch auf die niedrigen Werte (weit unter $100 / \mathrm{m}^{2}$ ) der Vorkriegszeit zurückzusinken.

\section{Die Sardine, Clupea pilchardus Walbaum}

1. Allgemeine Verbreitung

Das Verbreitungsgebiet der Sardine wird etwa von der 20 - und $10^{\circ}$ Isotherme der mittleren Jahrestemperatur begrenzt (FURNesTrn 1952) und umfaßt große Teile des Mittel- und Schwarzen Meeres sowie das atlantische Küstengebiet von Kap Blanco und den Azoren im Süden bis zur Keltischen See und dem Englischen Kanal im Norden. Hier wurde die Straße von Dover bis etwa zum Jahre 1930 anscheinend nicht überschritten, da für diese Zeit 
keine sicheren Nachweise von Sardinen in der südlichen Nordsee vorliegen. Gelegentlich traten sie dagegen an der Ostküste Schottlands, vor der TyneMündung, in den norwegischen Gewässern zwischen Bergen und Oslo und im Kattegat auf, doch immer nur in einzelnen oder wenigen Exemplaren. Erst in den 30er Jahren fing man sie häufiger in der Nordsee, Juni 1932 zusammen mit Heringen im Gebiet North Shields (Meex 1932) und Juni 1938 auch an der ostfriesischen Küste in Mengen bis zu $50 \mathrm{~kg}$ (Hass 1938). Eine weitere Zunahme des Auftretens von Sardinen in der Nordsee erfolgte 1946 und in den nächsten Jahren:

1946 Juli-Oktober. Norwegische Küste von Bergen bis in den Trondheim-Fjord. Häufig; meist ausgelaichte Tiere (Dons 1946)

1949 Mai und Juli. Ostfriesland, Dollart, Norddeich. In größeren Mengen (MEyer 1949),

1950 Oktober. Auf und südlich der Dogger Bank. Zwischen Heringen.

1951 Themse-Mündung, Lowestoft, Yarmouth. In ungewöhnlichen Mengen.

September. Westlich Schottland in der Minch.

Dezember. Firth of Forth. Etwa $42 \mathrm{~kg}$.

(Informationen 1951, 1952; RAE \& WILSON 1952).

Wie bei der Sardelle verlief also auch die Einwanderung der Sardine in die Nordsee in zwei Etappen, einer ersten in den auf 1930 folgenden Jahren und einer zweiten nach 1946.

\section{Laichverhältnisse}

Während der vielen von 1891 an bis zur Zeit des Zweiten Weltkricges von deutscher, englischer und holländischer Seite durchgeführten Fischbrutuntersuchungen wurden niemals Eier oder Larven der Sardine in der südlichen Nordsee beobachtet. Ihre nördlichsten Laichplätze lagen bisher in der Keltischen See (bis etwa 8- $9^{0} \mathrm{~W}$ ), vor der Küste von Cornwall und im Englischen Kanal. Hier reichte das Brutvorkommen bis zur Straße von Dover, diese aber nur ausnahmsweise einmal überschreitend - im Juni 1909 fand BuchananWollaston Sardinen-Eier bis zur Sandettié-Bank (Corbin 1950). Erst 1948 traten im Juni wiederum Sardinen-Eier ostwärts der Straße von Dover bei Sandettié-Bank, Goodwin-Feuerschiff, Bligh-Bank und Ost-Hinder-Bank auf (CorbiN), und in den folgenden Jahren konnten sie von uns auch in der südöstlichen Nordsee festgestellt werden.

Auf den Fahrten im Jahre 1948 (Mai, Juli und Oktober), die sich alle auf das nordfriesische Seegebiet beschränken, werden Sardinen-Eier noch nicht beobachtet. Sehr wahrscheinlich gehören aber die auf der Oktoberfahrt westlich des Lister Tiefs und vor der Elbe-Mündung gefangenen Clupeiden-Larven (Länge 29-37 mm, Zahl der Myomere 52-54) zur Sardine. Mit Sicherheit kann das Laichen der Sardine in der Deutschen Bucht erstmalig im Mai 1949 durch den Fang einiger Eier bei Amrum-Bank, Helgoland und südlich der Insel nachgewiesen werden. In größerer Menge treten Eier während der Sommerfahrt auf den westlichen Stationen auf; Larven werden auf dieser Fahrt nicht beobachtet:
3. Juli Osterems, $7 \mathrm{sm}$ nördl. Borkum
4. Juli $15 \mathrm{sm}$ nördlich
$9 \mathrm{Eier} / \mathrm{m}^{2}$
Salzgehalt $33,49^{\circ} \% 0$
Borkum und Norderney
4. Juli Westerems, $8 \mathrm{sm}$ nördl. Schiermonnikoog
$6-36 \quad$
1426 Eier in einem Knüppelnetzfang
$33,75-33,92$
über 33

Während der September-Fahrt (nordfries. Gebiet) fehlt Sardinen-Brut. 
1950 sind Anfang Mai bei Helgoland noch keine Eier anzutreffen. Im Juni finden wir sie dagegen vor der ostfriesischen Küste bis vor die Jade-WeserMündung, Larven darüber hinaus bis Helgoland und Amrum-Bank (Abb. 7). Das Vorkommen der Eier ist deutlich an das sogen. "Westliche Wasser" gebunden; die höchsten Werte, 273-276 je $\mathrm{m}^{2}$, liegen noch innerhalb der 34\%\%0Isohaline, und das äußerste Vorkommen von Eiern fällt mit dem Ende der nach Osten vorgeschobenen 33\%0-Zunge zusammen. Ebenso wird im Juli die

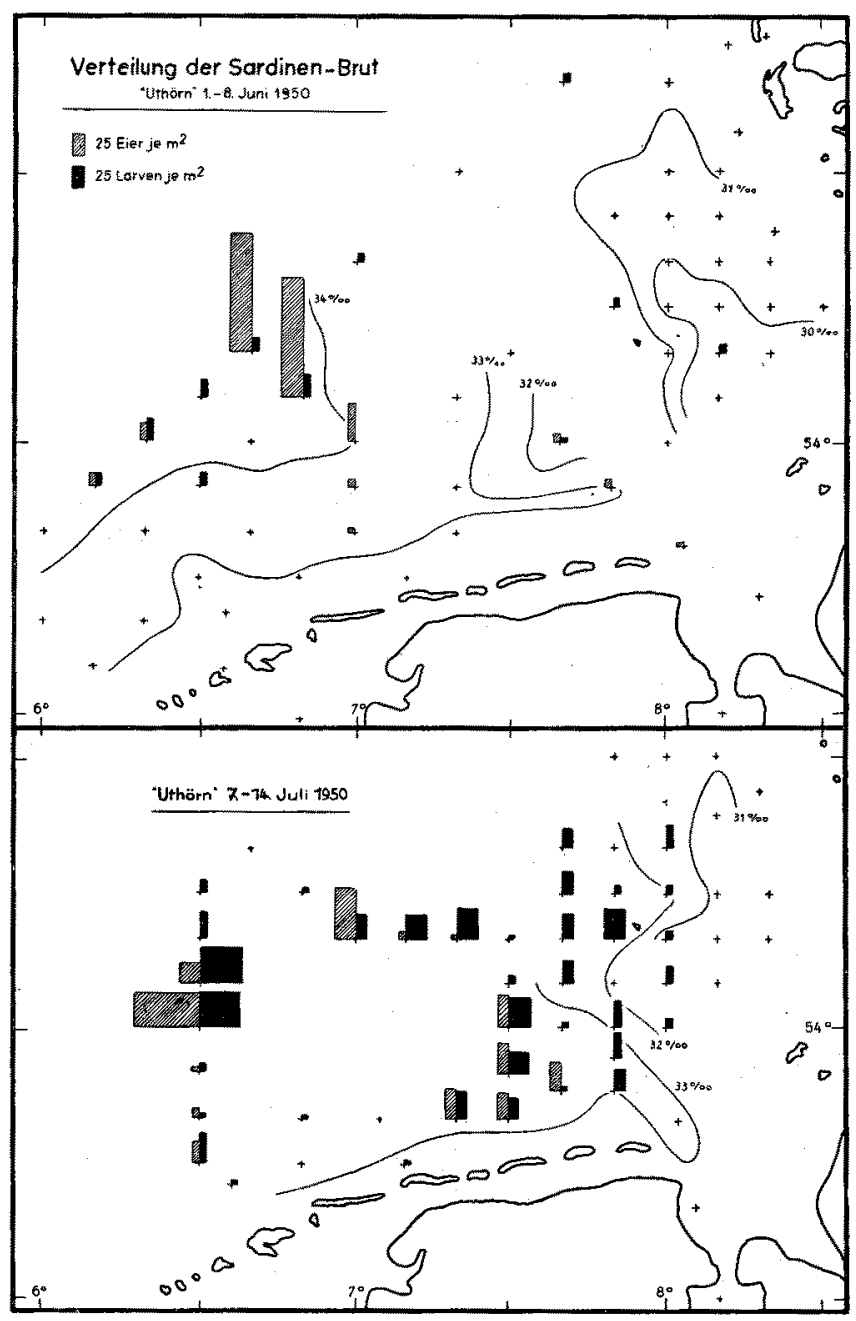

Abb. 7. Verteilung der Sardinen-Brut im Juni und Juli 1950

größte Brutdichte (120-234 Eier, 50-165 Larven je $\mathrm{m}^{2}$ ) nördlich BorkumNorderney bei einem Salzgehalt von 33,91-34,13\%o erreicht und die Ausbreitung der Eier im Osten wieder durch die 33\%o-Linie begrenzt. In späteren Fängen (August und November) aus dem nordfriesischen Gebiet fehlt Sardinen-Brut, desgleichen auch während der Frühjahrsfahrten (April bis Anfang Juni) 1951 im gleichen Gebiet. Besonders häufig ist sie dann aber während der beiden Sommerfahrten dieses Jahres (Abb. 5 und 6). Die Darstellung ihrer Verbreitung im Juni zeigt ein ausgedehntes Brutvorkommen (279 Eier, 
639 Larven je $\mathrm{m}^{2}$ ) zwischen Terschellinger Bank und Borkum Riff mit einem auffallenden Überwiegen von Larven auf den nördlichen, und von Eiern auf den südlichen Stationen. Durch eine nahezu brutleere Zone abgetrennt, folgt ein zweites, schwächeres Laichen nördlich Norderney (bis 75 Eier je $\mathrm{m}^{2}$ ), das sich aber bis zum Juli wesentlich verstärkt $\left(588 \mathrm{je} \mathrm{m}^{2}\right)$. Auf Grund dieser Verteilung darf angenommen werden, daß die Laichschwärme der Sardine, dem aus dem Kanal her kommenden "Westwasser" folgend, von West nach Ost fortschreitend ablaichen. Die großen, nordöstlich Terschellinger Bank angetroffenen Larvenmengen sind sicher von Laichplätzen hierher verdriftet, die vor der holländischen Küste und in der Flämischen Bucht zu suchen sind, so wie die von uns alljährlich im nordfriesischen Seegebiet beobachteten Larven von den Laichplätzen vor der west- und ostfriesischen Küste stammen.

Ende September ist das Laichen noch nicht beendet: am 30. September werden $20 \mathrm{sm}$ nordwestlich Helgoland noch 811 Eier, am 29. September auf einer Station $45 \mathrm{sm}$ westlich des Lister Tiefs 22 Eier je Knüppelnetz-Fang erbeutet $(\mathrm{Abb} .6)$. Auch diese Beobachtungen sind beweisend für die allmähliche Verlagerung des Laichens mit fortschreitender Jahreszeit nach Nordosten, denn auf keiner der Frühjahrs- und Sommerfahrten konnten Sardinen-Eier bisher so weit nördlich nachgewiesen werden.

1952 wurde die Sommerfahrt zwecks Feststellung der Grenzen der Brutverteilung nach See zu bis nahe an den Südostrand der Dogger-Bank ausgedehnt. Wie bereits aus den Beobachtungen der Vorjahre zu vermuten war, bleibt das Vorkommen von Sardinen-Eiern ganz an die Westwasser-Drift gebunden. Auf keiner Station des nördlich $54^{0} 15^{\prime} \mathrm{N}$ liegenden Gebietes, das durch massenhaftes Auftreten von Aglantha digitalis rosea und großen, geschlechtsreifen Exemplaren von Sagitta elegans als "nördliches Wasser" gekennzeichnet ist, werden Sardinen-Eier gefangen, Larven nur auf zwei Stationen in der Südwestecke des Gebietes. Erst von $54^{0} \mathrm{~N}$ an treten Eier in nach Süden zunehmender Dichte auf; Kernpunkte des Laichens liegen im Gebiet von Borkum Riff (maximal 375 Eier, 252 Larven je $\mathrm{m}^{2}$ ) und nördlich Norderney-Langeoog (648 Eier, 99 Larven je $\mathrm{m}^{2}$ ). Auch diesmal erweist sich das westliche Gebiet mit durchschnittlich 56 Eier, 76 Larven je $\mathrm{m}^{2}$ gegenüber dem östlichen mit im Durchschnitt 77 Eier, 23 Larven je $\mathrm{m}^{2}$ durch das Uberwiegen des Larven-Anteils als das „ältere“.

Das früheste Auftreten von Eiern tallt in diesem Jahre auf den 31. Mai (bei Helgoland); ältere Larven-Stadien werden noch am 12. November bei und südwestlich Helgoland angetroffen.

\section{Folgerungen aus der Verteilung der Sardinen-Brut}

Als Ergebnis unserer Untersuchungen über die Brutverteilung der Sardine folgt:

1. Seit mindestens 1949 , sehr wahrscheinlich bereits seit 1948, laicht neben der Sardelle jetzt auch die Sardine in größerem Umfange in der südlichen Nordsee.

2. Das Laichen ist deutlich an die aus dem Englischen Kanal kommende Westwasser-Drift mit dem relativ hohen Salzgehalt von über 33\% gebunden und rückt mit fortschreitender Jahreszeit allmählich nach Nordosten vor. Bereits im Juni werden vor den westfriesischen Inseln durchschnittlich mehr Larven als Eier gefangen, während diese im ostfriesischen Raume zur gleichen Zeit vorherrschend sind und im nordfriesischen Seegebiet 
überhaupt noch fehlen. Erst viel später, Ende September, treten Eier auch hier auf den am weitesten in See gelegenen Stationen auf. Daß es sich hierbei nicht etwa um bloße Verdriftung der Eier handelt, schließt deren kurze Entwicklungsdauer, die nach unseren Beobachtungen nur 3-4 Tage beträgt, und der Entwicklungsgrad der im September beobachteten Eier (fast durchweg auf allerjüngsten Stadien, noch ohne Embryo-Anlage) aus.

3. Gegenüber den Temperaturverhältnissen zeigt sich die Sardine dagegen weit unabhängiger, was sich besonders in der lang ausgedehnten Laichzeit bemerkbar macht. Der früheste Termin, an dem wir Sardinen-Eier, wenn auch in geringer Zahl (1-3 je Knüppelnetz-Fang), in der Deutschen Bucht feststellen konnten, ist der 11. Mai 1949, zu welcher Zeit die OberflächenTemperatur erst $7-10^{\circ}$ betrug. Und die spätesten Fänge stammen vom 30. September 1951, als auf einigen Stationen nordwestlich Helgoland und westlich des Lister Tiefs bei Oberflächen-Temperaturen von $15,7-16,2^{\circ}$ bis zu 811 Eier je Knüppelnetz-Fang erbeutet wurden. Es darf angenommen werden, daß das Laichen bis weit in den Oktober währt, da verhältnismäßig junge Larven (Länge 20-30 mm, Bauchflossen noch nicht oder eben erst angelegt) noch Mitte November in der Deutschen Bucht angetroffen werden. Zur Zeit des maximalen Vorkommens der Eier betragen die Temperaturen in unserem Gebiet $13-18,5^{\circ}$ und liegen somit etwas höher als die von FAGE für das Mittelmeer und atlantische Küstengebiet ermittelten optimalen Werte $\left(12-15^{\circ}\right)$.

4. Wie aus der folgenden Zusammenstellung der durchschnittlichen und maximalen Häufigkeit der Eier und Larven hervorgeht, hält sich die Brutdichte in allen Beobachtungsjahren etwa auf gleicher Höhe:

\begin{tabular}{|c|c|c|c|c|c|}
\hline & & \multicolumn{2}{|c|}{ Eier je $\mathrm{m}^{2}$} & \multicolumn{2}{|c|}{ Larven je $\mathrm{m}^{2}$} \\
\hline 1949 & Juli & 25 & 50 & & \\
\hline 1950 & Juni & 62 & 276 & 9 & 15 \\
\hline & Juli & 47 & 234 & 28 & 165 \\
\hline 1951 & Juni & 93 & 279 & 117 & 689 \\
\hline & Juli & 125 & 588 & 70 & 192 \\
\hline 1952 & Juli & 98 & 648 & 43 & 252 \\
\hline
\end{tabular}

Die für 1949 mitgeteilten Werte müssen außer Betracht bleiben, da in diesem Jahre das Laichgebiet der Sardine nur am Rande angeschnitten wurde. Ebensowenig kommt dem geringen Anstieg, der sich namentlich bei den Maximalwerten zeigt, eine Realität zu; er ist wohl allein auf die Erweiterung des Untersuchungsgebietes nach Westen hin während der späteren Jahre zurückzuführen.

\section{Diskussion der Ergebnisse}

\section{Massenwechsel}

Bei der Analyse von Bestandsveränderungen, die bei quantitativer Betrachtung zunächst als ein Massenwechsel erscheinen, sind je nach den Ursachen, durch die solche Veränderungen veranlaßt und in ihren Ausmaßen bestimmt werden, drei verschiedene Vorgänge zu unterscheiden:

Unter dem Begriff $\mathrm{Fluktuation}$ (und Gradation) werden solche Schwankungen in sich geschlossener Bestände vereinigt, deren Ursache in der 
Veränderlichkeit der natürlichen, biotischen und abiotischen Faktoren liegt. Es sind dies, wenn wir die Betrachtung auf den Massenwechsel bei Fischen einschränken: 1. Die. Größe der Brut-Erzeugung, die ihrerseits abhängig ist von der Zahl, dem Alter und der Größe der fortpflanzungsfähigen Tiere. 2. Die natürliche Zehrung, deren Größe in Beziehung steht zu den von der Natur gebotenen Lebensraumbedingungen (Milieu), zu der Art und Zahl der Feinde einschließlich der Krankheitserreger, zu dem Angebot geeigneter Nahrung und schließlich zu der Größe und Alterszusammensetzung des Bestandes selbst. Besonders in der Zehrung während der frühen Entwicklung, die größenmäßig im wesentlichen von dem Angebot geeigneter Nahrung während der kritischen Periode des Überganges von der Dotter-Ernährung zum aktiven Nahrungserwerb und von der Vernichtung durch Feinde abhängt, sieht man heute den die Fluktuationen ausschlaggebend bestimmenden Faktor. 3. Die als Wachstum sich äußernde Produktionsgröße, deren Schwankungen teils durch äußere Faktoren, vor allem durch das Nahrungsangebot und die Temperatur, teils durch den physiologischen Zustand der Tiere - Alter, erblich festgelegte Wüchsigkeit, - bestimmt werden. 4. Neuerdings muß auch noch der neben der Beute-Räuber-Beziehung bestehende ein- oder wechselseitige Einfluß der Organismen aufeinander durch Stoffwechel- und Ausscheidungsprodukte ("ectocrine", Lucas) als die Fluktuationen mitbestimmendes Moment in Betracht gezogen werden.

Den Fluktuationen als naturbedingten Massenschwankungen schließen sich die durch den Eingriff des Menschen bedingten Änderungen der Bestandsgröße an, die als Befischung zusammengefaßt werden können. Die Größe des als Befischung wirkenden Zehrungsfaktors wird aber nicht, wie bei der natürlichen Zehrung, ausschließlich durch die in der Natur gegebenen Verhältnisse bestimmt, sondern hängt maßgeblich von technischen, wirtschaftlichen und politischen Momenten ab.

Schließlich können Bestandsveränderungen innerhalb eines bestimmten Gebietes auch durch räumliche Verschiebungen der Verbreitungsareale, durch Trans- oder Regressionen verursacht werden, wobei die Größe des Gesamtbestandes einer Art unverändert bleiben kann.

Versuchen wir, den bei der Sardelle festgestellten Massenwechsel zu analysieren, so scheidet ein Einfluß durch Befischung aus, da in der Nordsee, abgesehen von der Zuider Zee, die Sardellen-Fischerei völlig bedeutungslos ist. Es fragt sich nun, ob das Anwachsen des Sardellen-Bestandes während der letzten Jahre im Sinne einer Fluktuation gedeutet werden kann.

Nach den holländischen Untersuchungen über die Sardelle der Zuider Zee weist diese Population ganz ungewöhnlich große Schwankungen auf, die Hoek (1912) damit zu begründen versuchte, daß hier, an der äußersten Grenze des Wohngebietes der Sardelle, die Lebensbedingungen während der kritischen, für die Fluktuationen entscheidenden Perioden, nämlich zur Zeit der $\mathrm{Ei}$-Ablage und der Larvenentwicklung, besonders starken Schwankungen ausgesetzt seien. Das Verhältnis zwischen schlechtestem und bestem Fangertrag in der Zuider Zee betrug für die Jahre 1875-1902 (nach HoEK) 1:190 und würde sich bei Berücksichtigung eines noch längeren Zeitraumes sogar auf 1:300 erweitern (schlechtester Fang $190832 \mathrm{t}$, bester $189010000 \mathrm{t}$ ).

Eine entsprechende Berechnung der Bestandsschwankungen für die südöstliche Nordsee auf Grund der Fangerträge hat lediglich bedingten Wert, da hier eine Sardellen-Fischerei nur unmittelbar an der Küste ausgeübt wird, so 
daß der auf See bleibende Bestand, der ja nach unseren Beobachtungen über die Brut-Verteilung besonders stark angewachsen war, überhaupt nicht erfaßt wird. Nach den Anlandungen der ostfriesischen Küstenfischerei ergeben sich von 1920-1952 folgende Veränderungen der Bestandsgröße:

\begin{tabular}{|c|c|c|c|c|}
\hline & $\begin{array}{c}\text { durchschnittlicher } \\
t\end{array}$ & $\underset{\substack{\text { Jainster } \\
t}}{\text { Jahrese }}$ & $\underset{\substack{\text { größter } \\
t}}{ }$ & kleinster/größter \\
\hline $1920-1929$ & 3,4 & $0,4(1920,1924)$ & $12,0(1926)$ & $1 / 30$ \\
\hline $1930-1947$ & 24,5 & $1,0(1933)$ & $100,5(1931)$ & $1 / 100$ \\
\hline $1948-1952$ & 58,5 & $26,6(1952)$ & $109,0(1949)$ & $1 / 4$ \\
\hline
\end{tabular}

Und als Maß für die Schwankungsbreite während des gesamten Zeitraumes ein Verhältnis

$$
\frac{\text { geringster Jahresertrag }}{\text { größter Jahresertrag }}=\frac{0,4 \mathrm{t}(1920,1924)}{109,0 \mathrm{t}(1949)}=\frac{1}{127},
$$

das in der Größenordnung etwa den in der Zuider Zee beobachteten Schwankungen entspricht. Ein wesentlich größerer Unterschied ergibt sich, wenn der Vergleich auf der Basis der Laich-Produktion durchgeführt wird.

Als relatives Maß für die jeweilige Größe eines Bestandes läßt sich das Produkt aus der durchschnittlichen Ei-Dichte während der Hochzeit des Laichens und der Fläche des Laichgebietes verwenden, das ist die Gesamtmenge der durchschnittlich zu einem Zeitpunkt vorhandenen Eier. Für die südliche Nordsee ergeben sich hiernach folgende Werte:

\begin{tabular}{lcccc} 
& & $\begin{array}{c}\mathbf{a} \\
\text { durchschnittliche } \\
\text { Häufigkeit der } \\
\text { Eier je m }\end{array}$ & $\begin{array}{c}\text { L } \\
\text { Laichareal } \\
\text { in } \mathrm{km}^{2}\end{array}$ & $\begin{array}{c}\mathbf{a} \times \mathbf{b} \\
\text { Laichproduktion }\end{array}$ \\
\hline südöstliche Nordsce & $1902-1929$ & 1 & 200 & $0,2 \times 10^{9}$ \\
& $1930-1939$ & 10 & 500 & $5 \times 10^{9}$ \\
Zuider Zee & $1948-1952$ & 400 & 3000 & $1200 \times 10^{8}$ \\
& $1906-1912$ & 200 & 1000 & $200 \times 10^{8}$
\end{tabular}

Für die früberen Jahre, 1902-1939, läßt sich die Größe der Laih-Areale in der südöstlichen Nordsee nur annähernd bestimmen, da damals die Sardellen-Brut nicht über größere, zusammenhängende Gebiete verteilt war, sondern immer nur auf wenigen, vereinzelten Statıonen angetroffen wurde. Die für 1948-1952 angegebenen Werte wurden nach der S. 190 angeführten Ubersicht unter Abrundung berechnet, diejenigen für die Zuider Zee nach Angaben über die dortigen Laich-Verhältnisse bei REdeke und Havinga.

Nach der Laich-Produktion zu urteilen, hat sich also der Sardellen-Bestand in der südöstlichen Nordsee von 1902/29 bis 1948/52 auf das rund 6000fache vergrößert und ist auch beträchtlich größer als die Zuider Zee-Population geworden, eine Änderung, die sich keineswegs noch im Sinne einer Fluktuation verstehen läßt. Gegen eine solche Deutung spricht außerdem der einsinnige Verlauf der Bestandsänderung, der sich sowohl in den Fangerträgen wie auch in der Laich-Produktion als eine fortschreitende Zunahme offenbart.

Es fragt sich nun, aus welcher Quelle diese Zunahme des Sardellen-Bestandes stammt. Folgende Erklärungen sind möglich:

1. Der jetzt in der südöstlichen Nordsee laichende Bestand ist der unmittelbare Nachfolger der ehemaligen Zuider Zee-Population. 
2. Die Bestandszunahme in der Nordsee ist auf eine vermehrte Einwanderung von Teilen eines atlantischen Mutterbestandes zurückzuführen.

Durch vergleichende Untersuchungen der Ertragsschwankungen in der Nordsee, den atlantischen Küstengebieten und im Mittelmeer versuchte HoEk die Frage zu klären, wie weit die einzelnen Populationen voneinander abhängig seien. Aus dem Fehlen synchroner Korrelationen, positiver oder negativer Art, zwischen den Ertragsschwankungen in den verschiedenen Gebieten glaubt Новк annehmen zu dürfen, daß die Sardelle in der Zuider Zee einen sich selbst erhaltenden, eigengesetzlichen Bestand bilde. Aus ähnlichen Erwägungen spricht auch HAviNGA (1950) der Schelde-Population eine gewisse Autonomie zu. Nachdem durch den Abschluß der Zuider Zee die Sardelle ihres bedeutendsten Laichplatzes in der Nordsee beraubt worden war, lag es zunächst nahe, anzunehmen, daß der Zuider Zee-Bestand sich notgedrungen in der südöstlichen Nordsee einen neuen Laichplatz habe suchen müssen. Gegen eine solche Annahme spricht aber einmal die Parallelität der Bestandsschwankungen bei den verschiedenen Nordsee-Populationen der Sardelle schon vor dem Abschluß der Zuider Zee, ferner der historische Verlauf während und kurz nach der Eindeichung, vor allem aber die gleichzeitig erfolgende Bestandszunahme bei verschiedenen anderen Fischen.

Das erste Anwachsen des Sardellen-Bestandes erfolgte, wie wir sahen (S. 178), bereits 1930. Damals stiegen in der Zuider Zee die Erträge weit über den langjährigen Durchschnitt:

$\begin{array}{llc}\text { Durchschnittsertrag } & 1843-1929 & 1338 \mathbf{t} \\ \text { Jahreserträge } & 1930 & 5338 \\ & 1931 & 3478 \\ & 1932 & 378,\end{array}$

zur gleichen Zeit machte sich aber auch in der ganzen übrigen Nordsee, ja sogar bis in die mittlere Ostsee hinein die Zunahme der Sardelle bemerkbar (vergl. Abb. 1 u. S. 179).

Kurz bevor am 28. Mai 1932 der Abschlußdeich der Zuider Zee vollendet wurde, konnte noch ein großer Teil der eben angekommenen Sardellen ( „a rather normal migration" HavingA) in das Becken einwandern, war dadurch aber gefangen. Nur den Larven, die aus dem auch 1932 noch erfolgreichen Laichen hervorgingen, gelang es offensichtlich, durch Schleusen und durch Löcher im Damm zu einem großen Teil wieder zu entkommen. Ihnen schreibt HAvinga die auch 1933 wieder übernormalen Fänge im holländischen Wattenmeer zu. Dagegen hatte der Abschluß der Zuider Zee keinen merkbaren Einfluß auf die Fänge in den anderen Gebieten, in der Ooster-Schelde und an der ostfriesischen Küste, vielmehr sanken hier die Erträge schon 1932 wieder auf die Norm zurück. Erst 6-10 Jahre später, 1938 und 1941/42, traten erneut überdurchschnittliche Fänge ein, 1948/52 von einem zweiten noch stärkeren Zuwachs der Bestände gefolgt (Abb. 1). Zu diesen Zeitpunkten waren aber, da die Sardelle höchstens 2-3 Jahre alt wird, auch die letzten, noch in der Zuider Zee geborenen Tiere bereits weggestorben.

Gleichzeitig mit dem letzten, 1948 beginnenden Zuwachs der Sardellenbestandes erscheint erstmalig die Sardine als Laichfisch in der südlichen Nordsee und nehmen auch die Bestände einiger anderer, mediterran-atlantischer Arten hier zu, wie der folgende Vergleich der Brut-Dichten zeigt: 
In den einzelnen Jahren beobachtete Höchstwerte für die Eier je $\mathrm{m}^{2}$

\begin{tabular}{lcc} 
& $1904-1930$ & $1949-1952$ \\
\hline $\begin{array}{l}\text { Scezunge (Solea valgaris) } \\
\text { südöstliche Nordsee }\end{array}$ & $10-35$ & $100-276$ \\
$\begin{array}{l}\text { Bastardmakrele (Caranx } \\
\text { trachurus) } \\
\text { südöstliche Nordsee }\end{array}$ & $10-77$ & $500-850$ \\
Flämische Bucht & $50-460$ & einmal 2520 (querab Norderney) \\
$\begin{array}{l}\text { Makrele (Scomber scombrts) } \\
\text { südliche Nordsee }\end{array}$ & einmal 3650 (Maas Fsch.) & $100-370$ \\
& $40-126$ & einmal 1716 (Westteil Austerngrund)
\end{tabular}

Diesen Beispielen kann man für die Nordsee noch die Steigerung der Seehecht- und Thunfisch-Fänge und das häufige Auftreten von Brachsenmakrelen (Brama rayi) in den Jahren nach dem letzten Kriege hinzufügen.

Als Antwort auf die oben gestellten Fragen ergibt sich hieraus: Weder die einzelnen Teilpopulationen (Scheldemündung, Zuider Zee, Deutsche Bucht) noch die Sardellen-Bevölkerung der Nordsee in ihrer Gesamtheit sind unabhängig und autonom in dem Sinne, daß die Erhaltung ihrer Bestände, unter Voraussetzung der Geburtsortstreue, allein durch die eigene Nachkommenschaft gesichert wird und daß somit die Schwankungen ihrer Bestandsgröße in erster Linie auf solche der Entwicklungsmöglichkeit und Zehrung ihrer Nachkommen zurückzuführen sind, sondern es muß angenommen werden, daß die Populationen wenigstens zeitweise einen wesentlichen Zuwachs durch $\mathrm{Zu}$ wanderung von Fischen aus einem atlantischen Mutterbestand erfahren. In diese Richtung deutet auch ein der Einwanderung der Sardine in die Nordsee entsprechender gleichzeitiger Rückgang der Sardinen-Erträge an der Atlantikküste von Spanien und Portugal. Wenn Hoek bei der Sardelle keine solche negative Korrelation zwischen den Erträgen in der Zuider Zee und den atlantischen Gebieten nachweisen konnte und daraus auf die Unabhängigkeit der Zuider Zee-Population schloß, so beruht dies wohl auf der von ihm selbst hervorgehobenen Unsicherheit der benutzten, statistischen Unterlagen, in denen die Erträge von Sardelle und Sprott nicht immer getrennt aufgeführt wurden. Sehr wahrscheinlich sind auch die früher in der Zuider Zee beobachteten ungewöhnlich hohen Ertragsschwankungen nicht im Sinne von Fluktuationen zu deuten, sondern beruhen auf zeitweiligen Verstärkungen durch Transgressionen des atlantischen Bestandes.

Man muß sich diese Art des Massenwechsels als räumliche Verschiebungen des ganzen Verbreitungsareals oder doch großer Teile desselben vorstellen, wobei es in den einen Gebieten zur Verarmung oder gar zum völligen Erlöschen von Beständen kommt (Regressionen), an anderen Stellen dagegen zu einer Zunahme oder zu neuen Ansiedelungen (Transgressionen). Verursacht wird das ganze Geschehen ohne Zweifel durch einen Wechsel der hydrographischen Verhältnisse, in erster Linie der Strömungs- und Temperaturverhältnisse. Aus der Zunahme bzw. Einwanderung mediterran-atlantischer Arten in unserem Gebiet folgt, daß sich hier das Milieu in Richtung auf die im Hauptverbreitungsgebiet dieser Arten herrschenden Verhältnisse verändert hat. 


\section{Beziehungen zur Klimaänderung}

Eine solche Veränderung der Milieufaktoren findet nachweislich seit mehreren Dezennien im Zusammenhang mit der Klimaänderung statt, deren Beginn in die Mitte des vorigen Jahrhunderts, vielleicht schon an dessen Anfang, zu setzen ist (AhLmann 1949, Wagner 1940). Allgemein äußert sich diese Klimaänderung in einer Zunahme des meridionalen Druckgefälles und einer dadurch bedingten Verstärkung der Zirkulation, deren Folge auf der nördlichen Halbkugel ein vermehrter Transport von Luft und Wasser aus westlichen und südlichen Richtungen und damit auch von Wärme nach höheren Breiten ist.

Die weiteren Auswirkungen der Klimaänderung traten zuerst in der unbelebten Natur in Erscheinung: Rückgang des Eises und dadurch Verbesserung der Schiffahrtsverhältnisse in den polaren Gebieten, Rückzug der Gletscher in allen Erdteilen, gleichsinnig fortschreitende Änderung der Pegelstände, der Häufigkeiten von Sturmfluten u.a. In der Biosphäre wurden entsprechende Folgeerscheinungen erst später bemerkbar, vorwiegend in den nördlichen $\mathrm{Ge}$ bieten. Die Gründe hierfür liegen vielleicht darin, daß die Organismen als gleichsam besser gepufferte Systeme im allgemeinen schwächer und langsamer, in manchen Fällen vielleicht auch erst nach Überschreiten eines gewissen Schwellenwertes auf Veränderungen ihres Lebensraumes reagieren. Die Feststellung eines Einflusses der Klimaänderung wird außerdem erschwert durch den Mangel an zeitlich weiter zurückreichenden, vergleichbaren Beobachtungsreihen über die Zusammensetzung und Größe der Organismenbestände, so daß deren Veränderungen oft erst dann festgestellt werden können, wenn sie ein beträchtliches Ausmaß erreicht haben.

Da die Erwärmung in den Gebieten höherer Breite frühzeitiger eintrat und von stärkerem Ausmaße war als in geringeren Breiten, machte sich der Einfluß der Klimaänderung vorwiegend in den Tierbeständen der nördlichen Meere bemerkbar, wo er besonders große Folgen für die praktische Fischerei hatte:

Barents See:

1905 Beginn der praktischen Fischerei. Bei Spitzbergen fand „Olga“ 1905 noch keine Fische 1930-1933 Erweiterung des Fanggebietes bis auf die östlichen Bänke und 1937 bis Spitzbergen.

Murman Küste:

1920-1930 Jahresertrag der Heringsfischerei $60-1000 \mathrm{t}$, durchschnittlich $345 \mathrm{t}$

1931-1933 23000-68 $000 \mathrm{t}$, durchschnittlich über $40000 \mathrm{t}$.

West Grönland:

1911-1947 Kabeljau-Erträge von 18 auf $14900 \mathrm{t}$ jährlich ansteigend, seit 1929 treten auch Shellfische auf.

Ost Grönland:

Vor 1912 keine Fischerei auf Kabeljau,

1912-1923 Kabeljau häufiger, seit 1930 Kabeljau alljährlich in fangwürdigen Mengen.

Is land-Fa röer:

Seit etwa 1925 sind hier 11 Fischarten neu zugewandert und 14 Gast-Fische häufiger geworden.

Ab etwa 1937 wird Kabeljau- und Schellfisch-Brut auch an der Nordküste Islands beobachtet.

Westteil des Englischen Kanals:

Seit 1980/31 ständiger Rückgang der Häufigkeit der Jungfische (außer Clupeiden), auch von wirtschaftlich nicht genutzten Arten. 
Nordsee:

1927, 1949 häufigeres Vorkommen von Gast-Arten (Brachsenmakrele, Brama rayi), Aufblühen des Thunfisch-Fanges.

1946, 1947, 1952 Rekordfänge von Seehecht (Merluccius vulgaris).

Vielleicht steht im gleichen Zusammenhang auch das Verschwinden des Schellfisches aus der südlichen Nordsee (Rückgang seit Ende des vorigen Jahrhunderts) sowie das Erlöschen des Rochen-Fanges an der deutschen Küste.

Ostsee:

Seit 1930 Rückgang der Plattfisch-Erträge, Zunahme der Dorsch- und Herings-Fänge.

1932 Caranx in ungewöhnlicher Menge auftretend.

1932-1934, 1937 häufigeres Vorkommen von Drepanopsetta und deren Brut in der Belt-See und westlichen Ostsee.

1938 Eier und Larven der Makrele in größerer Menge in der westlichen Ostsee beobachtet, dort früher nur einmal, 1926, gefunden.

(Berg 1935; Brandes 1952; Fridriksson 1949; Hansen 1949; Heegaard 1947; Jensen 1948;

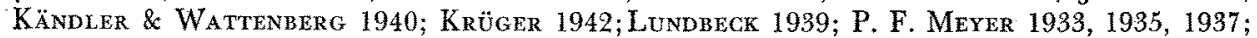
A. Meyer 1951; Russell 1940, 1947; TÅnXNg 1949).

Auf Grund unserer Beobachtungen, wonach sich die Bestandsänderungen in der südlichen Nordsee nicht stetig, sondern in einzelnen Etappen vollzogen haben - auch die oben angeführten Beispiele für die Vorgänge in anderen Gebieten deuten in dieser Richtung -, muß angenommen werden, daß die Anderung der klimatischen Faktoren in einzelnen Wellen (Schmauss spricht für die Jahrhundertwende von einer "Klima-Verwerfung") verlaufen ist, deren Intensität aber mit der Zeit zugenommen hat. Die letzten Wellen setzten 1930 und, in besonderer Stärke, um 1948 ein und äußerten sich in unserem Gebiet in einer Zunahme der Laichbestände der mediterran-atlantischen Arten. Für den Sardellen-Bestand zeichnet sich schon 1890 eine Welle besonders starker Bestandsvergrößerung ab, welches Jahr in der Zuider Zee den größten Fang ergab, fast $10000 \mathrm{t}$. Zur gleichen Zeit wuchs auch im übrigen Nordseegebiet der Sardellen-Bestand erheblich an: an der ostfriesischen Küste (Dollart bis Norddeich) wurden 1889 und 189015000 bzw. $16000 \mathrm{~kg}$ Sardellen gefangen, das ist das Dreifache des Durchschnittes der Jahre 1867-1907 (rund $5000 \mathrm{~kg}$ ), und im Juli 1891 wurde erstmalig der Nachweis ungewöhnlich großer Mengen von Sardellen-Eiern in der offenen See erbracht (S. 180). Vielleicht ist es auch nicht nur eine Folge des Aufblühens der Dampfer-Fischerei, wenn in den gleichen Jahren der starke Rückgang des Schellfisches in der südlichen Nordsee beginnt, der jetzt zum völligen Verschwinden des Fisches aus diesem Gebiet geführt hat.

Am Beginn der einzelnen Wellen der großen Bestandsveränderungen scheinen besondere Verhältnisse der Versetzung der Wasserkörper, Transgressionen (LE Danols 1928), „submarine eagres" (COOPER 1952) zu herrschen, durch die. Teile der atlantischen Populationen entweder mehr passiv, wie es Cooper für das zeitweilig häufige Auftreten des Eberfisches Capros aper, bei Plymouth annimmt, oder indirekt durch eine Veränderung des die Wanderrichtung bestimmenden Orientierungsfeldes (vergl. Hoeks Hypothese über die Bestimmung des Wanderweges der im Frühjahr durch den Englischen Kanal ziehenden Sardellen durch die besondere Temperaturverteilung, S. 177) in andere Gebiete geführt werden, in denen sich im Verlauf der allgemeinen Erwärmung die Milieuverhältnisse günstig für die Fortpflanzung und Entwicklung der betreffenden Arten gestaltet haben.

Über den zukünftigen Verlauf der Klimaänderung läßt sich auf Grund der biologischen Feststellungen nur soviel aussagen, daß sich ihr Einfluß bis in 
die neueste Zeit in stufenweise ansteigendem Maße bemerkbar macht und die bisher stärksten Auswirkungen gerade in die allerletzten Jahre (1948/52) fallen. Dagegen muß die Frage, wie lange die Entwicklung in gleicher Richtung weiterläuft, noch offen bleiben.

\section{Zusammenfassung}

Aus den in den Nachkriegsjahren durchgeführten Untersuchungen über die Verbreitung und Häufigkeit der Fischbrut während der Sommermonate ergibt sich, daß seit 1948 die Sardelle, deren Hauptlaichgebiet bisher in der Zuider Zee lag, in großem Umfange in der südöstlichen Nordsee laicht und einen bedeutenden Anstieg ihres Bestandes erfahren hat. Von der gleichen Zeit an tritt auch die Sardine, die früher nur sporadisch an der Südwestküste von Norwegen und Schweden erschien, als Laichfisch in der südlichen Nordsee auf. Das Laichen der Sardine ist streng an das durch den Kanal in die Nordsee setzende "westliche Wasser" gebunden.

Diese Bestandsveränderungen, die gleichzeitig auch bei einigen anderen mediterran-atlantischen Arten zu erkennen sind, lassen sich nicht im Sinne von Fluktuationen deuten, sondern sind die Folge von räumlichen Verschiebungen der Verbreitungsareale, die ihre Ursache in dem im Laufe der Klimaänderung eingetretenen Wechsel der hydrographischen Verhältnisse haben. Aus dem stufenweise erfolgten Anstieg der Fangerträge und der Brutdichten ist zu vermuten, daß auch die Klimaänderung nicht stetig, sondern in einzelnen Wellen verläuft. Aus den biologischen Beobachtungen ergibt sich ferner, daß die Erwärmung im gleichen Sinne bis in die letzten Jahre weiter fortgeschritten ist.

\section{Literatur.}

Ahlmann, H. W. 1949. Climatic Changes in the Artic in Relation to Plants and Animals. Introductory Address. Rapp. Proc.-Verb. Réun., Cons. Perm. Intern. Explor. Mer 125.

A urich, H. J. 1940. Die Verbreitung der pelagischen Fischbrut in der südlichen Nordsee während der Frühjahrsfahrten' 1926-1937. Helgol. Wiss. Meeresunters. 2.

B a hr, K1. 1933. Sardelle vor der ostpreußischen Samlandküste. Mitt. Deutsch. Seefisch. Ver. 49.

Berg, L. S. 1935. Rezente Klimaschwankungen und ihr Einfluß auf die geograph. Verbreitung der Seefische. Zoogeogr. 3.

Boeke, J. 1906. Eier und Jugendformen von Fischen der südlichen Nordsee. Verh. Rijksinst. Onderz. Zee 1.

Brandes, C. H. 1952. Über das Auftreten von Brachsenmakrelen in den nordeuropäischen Gewässern. Veröff. Inst. Meeresf. Bremerhaven 1.

Bückmann, A. 1929. Die Methodik fischereibiologischer Untersuchungen an Meeresfischen. Handb. biol. Arbeitsmeth. Abt. 9, Teil 6.

Cooper, L. H. N. 1952. The Boar Fish, Capros aper, as a possible biological indicator of water movement. J. Mar. Biol. Ass. 31.

Corbin, P. G. 1950. Records of pilchard spawning in the English Channel. J. Mar. Biol. Ass. 29.

Cunningham, J. T. 1895. The Migration of the Anchovy. J. Mar. Biol. Ass. 3.

- 1896. The Natural History of the Marketable Marine Fishes of the British Islands. London 1896.

Danois, E. le 1928. Les transgressions atlantiques et leurs conséquences biologiques. Rapp. Proc.-Verb. Réun., Cons. Perm. Intern. Explor. Mer 165.

Dons, C. 1946. Clupea pilchardus in Norway. Kgl. Norske Vidensk. Selsk. Forh. 19.

Ehrenbaum, E. 1892a. Die Sardelle. Deutsch. Fisch. Ver., Mitt. Sekt. Küsten- u. Hochseefischerei. 1892. Sonderbeilage. 
- 1892b. Bericht über die von der Sektion f. Küsten- und Hochseefischerei im Mai und Juni 1892 veranstaltete Versuchsfischerei auf der Unterems. Deutsch. Fisch. Ver., Mitt. Sekt. Küsten- u. Hochseefisch. 1892.

- 1908. Über Eier und Jugendformen der Seezunge und anderer im Frühjahr laichender Fische der Nordsee. Wiss. Meeresunters., N.F. Abt. Helgoland 8.

- 1932. Sardellen in der Deutschen Bucht. Der Fischerbote 24.

Fage, L. 1911. Recherches sur la Biologie de 1'Anchois. Annal. Inst. Océanogr. 2.

- 1920. Engraulidae, Clupeidae. Rep. Danish Oceanogr. Exped. 1908-10 to the Mediterranean and Adjacent Seas. 2, A 9.

Fischer, E. 1935. Die Arbeiten der Deutschen Wissenschaftlichen Kommission, Abt. Deutscher Seefischerei-Verein 1930-1933. Ber. Deutsch. Wiss. Komm. Meeresf. 7.

Fridriksson, A. 1949. Boreo-tended Changes in the Marine Vertebrate Fauna of Iceland during the last 25 years. Rapp. Proc.-Verb. Réun., Cons. Perm. Intern. Explor. Mer 125.

Furnestin, J. 1952. Biologie des Clupéidés Méditerranéens. Vie et Milieu, Suppl. 2.

Furnestin, J. u. R. Coupe 1950. Les Caractéristiques morpholog. des Anchois du Maroc. Journ. d. Cons., Cons. Perm. Intern. Explor. Mer 16.

Giudice, P. Lo 1911. Ancora sulle diverse Razze Locali di Acciughe, Rev. mens. Pesca e Idrobiol. 6.

Gourret, P. 1897. Les Etangs Saumâtres du Midi de la France et leurs Pêcheries. Annal. Mus. Hist. Nat. Marseille, Zool. 5.

Hagmei er, A. 1939. Die Arbeiten der Biolog. Anstalt auf Helgoland. Ber. Deutsch. Wiss. Komm. Meeresf. 9.

Hansen, Paul M. 1949. Studies on the Biology of the Cod in Greenland Waters. Rapp. Proc.-Verb. Réun., Cons. Perm. Intern. Explor. Mer 123.

Hass, 1938. Die diesjährige Sardellenfischerei in der Unterems und im ostfriesischen Wattenmeer. Die Deutsche Fischwirtschaft 1.

Having a, B. 1950. The Anchovy in the Dutch Waters after the Enclosure of the Zuidersea. Rapp. Proc.-Verb. Réun., Cons. Perm. Intern. Explor. Mer 126.

Heegaard, P. 1947. Investigations on the Proceeding Season and the Quantities of Eggs of the Food-Fishes of the Kattegat and the Northern Belt Sea 1929-1949. Medd. Komm. Danmàrks Fiskeri-og Havundersøg., Ser. Fiskeri 11.

Heincke, F. u. E. Ehrenbaum 1900. Eier und Larven von Fischen der Deutschen Bucht II. Wiss. Meeresunters., N.F. Abt. Helgoland 3.

Hoek, P. P. C. 1912. Lès Clupéides (Le Hareng Excepté) et leurs Migrations. Rapp. Proc.Verb. Réun., Cons. Perm. Intern. Explor. Mer 14.

Holt, E.W.L. 1897/99. Observations et recherches sur les oeufs flottants de Téléostéens faites dans le Golfe de Marseille. Recherches sur la reproduction des poissons osseux, principalement dans le Golfe de Marseille. Annal. Mus. Hist. Nat. Marseille, Zool. 5.

In formation en über die Fischwirtschaft des Auslandes. 1951 u. 1952. 1 und 2 (Hamburg).

Jenkins, J. T. 1925. The Fishes of the British Isles. London 1925.

Jensen, A.S. 1948. Contributions to the Ichthyofauna of Greenland. Spolia Zool. Mus. Hauniensis 9

Kändler, R. u. H. Wattenberg 1940. Einige Ergebnisse der Untersuchungsfahrten mit dem Reichsforschungsdampfer "Poseidon" in der westlichen Ostsee 1938. Ber. Deutsch. Wiss. Komm. Meeresf. 9.

Krüger, K. 1942. Enneutes Auftreten der Scharbzunge, Drepanopsetta platessoides, in der westlichen Ostsee. Kieler Meeresf. 4.

Legendre, R. 1934. La Faune Pélagique de l'Atlantique au Large du Golfe de Gascogne. Annal. Inst. Océanogr, 14.

Lundbeck, J. 1939. Veränderungen der Fischerei in den nordosteuropäischen Gewässern während der letzten zehn Jahre. Deutsche Fischerei-Rundschau 1939, 2.

McIntosh u. Masterman 1897. The Life Histories of the British Marine Food Fishes. London 1897.

Marion, M. A.F. 1889. Notes sur l'Anchois. Annal. Mus. Hist. Nat. Marseille Zool. 3.

Me ek, A. 1932. Faunistic Notes. Dove Mar. Lab., Rep. N. S. 21.

Meyer, A. 1951. Die erste Deutsche Sardinenfischerei. Fischereiwelt 3.

Meyer, P. F. 1930. Der Sardellenfang im Jadebusen. Mitt. Deutsch. Seefisch. Ver. 46.

- 1932. Beobachtungen über das Auftreten von Sardellenschwärmen im Jadebusen 1931. Der Fischerbote 24.

- 1933. Uber das Vorkommen von Sardellenschwärmen und von Bastardmakrelen in der Ostsee. Mitt. Deutsch. Seefisch. Ver. 49. 
- 1935. Massenauftreten der Scharbzungen in der Ostsee. Z. f. Fischerei 1935.

- 1937. Drepanopsetta-Besiedlung der westlichen Ostsee; eine Folge von Larvenverfrachtungen im Jahre 1930. Rapp. Proc.-Verb. Réun., Cons. Perm. Intern. Explor. Mer 102. 1949. Sardinen an der ostfriesischen Küste. Fischereiwelt 1.

Mielck, W. u. C. Künne 1935. Fischbrut- und Plankton-Untersuchungen auf dem Reichsforschungsdampfer "Poseidon" in der Ostsee. Wiss. Meeresunters, N. F. Abt. Helgoland 19

Rae, B. B. u. E. Wils on 1952. Records of rare Fishes. Annal. Biol., Cons. Perm. Intern. Explor. Mer 8.

Redeke, H. O. 1913/19. Bijdragen tot de Kennis van de teelt der Ansjovis in de Zuiderzee. Rapp. en Verh., Rijksinst. voor Visscherijonderzoek 1.

- 1916. Zur Naturgeschichte der Sardelle. Mitt. Deutsch. Seefisch. Ver. 32.

Russe11, F. S. 1940, 1947. On the Seasonal Abundance of Young Fish. VII und VIII. J. Mar. Biol. Ass. 24, 26.

Taning, A. V. 1949. On Changes in the Marine Fauna of the North-Western Atlantic Area with special reference to Greenland. Rapp. Proc.-Verb. Réun., Cons. Perm. Intern. Explor. Mer'125.

Tesch, J. J. 1909. Eier und Larven einiger im Frühjahr laichender Fische besonders der südlichen Nordsee. Verh. Rijksinst. Onderzoek der Zee 2.

- 1913/19. Weitere Untersuchungen über das Laichen einiger Nutzfische besonders der südlichen Nordsee. Rapp. en Verh. Rijksinst. vor Visscherijonderzoek 1.

Wagner, A. 1940. Klimaänderungen und Klimaschwankungen. Braunschweig. 